\title{
Gabriel Songel
}

Gabriel Songel es Catedrático de Diseño en la Universitat Politècnica de València, donde ha sido profesor académico en Diseño Industrial desde 1988. Después de asistir al Máster en Diseño Industrial en el Instituto Europeo de Diseño de Milán (1985-1987) obtuvo su doctorado en Metodología de Diseño en la Escuela de Ingeniería de la UPV en 1991.

Fue uno de los miembros fundadores del Grupo de Investigación y Gestión de Diseño de la UPV en 1991, cuando se comenzó a realizar el análisis de Tendencias de Diseño para diferentes instituciones y empresas. Socio fundador de Innoarea Design Consulting S.L., una Empresa de Base Tecnológica Spinoff de la UPV creada en 2008. Ha sido comisario de varias exposiciones nacionales sobre diseño e innovación.

Diseñador de más de 120 productos desde parques infantiles, juguetes, juegos y muebles para niños. Gabriel Songel ha realizado la primera tesis española en Biónica, leída en Abril de 1991 en la Universidad Politècnica de València, cuyo título es "Estudio metodológico de la Biónica aplicada al diseño industrial", dirigida por Eliseo Gómez Senent.

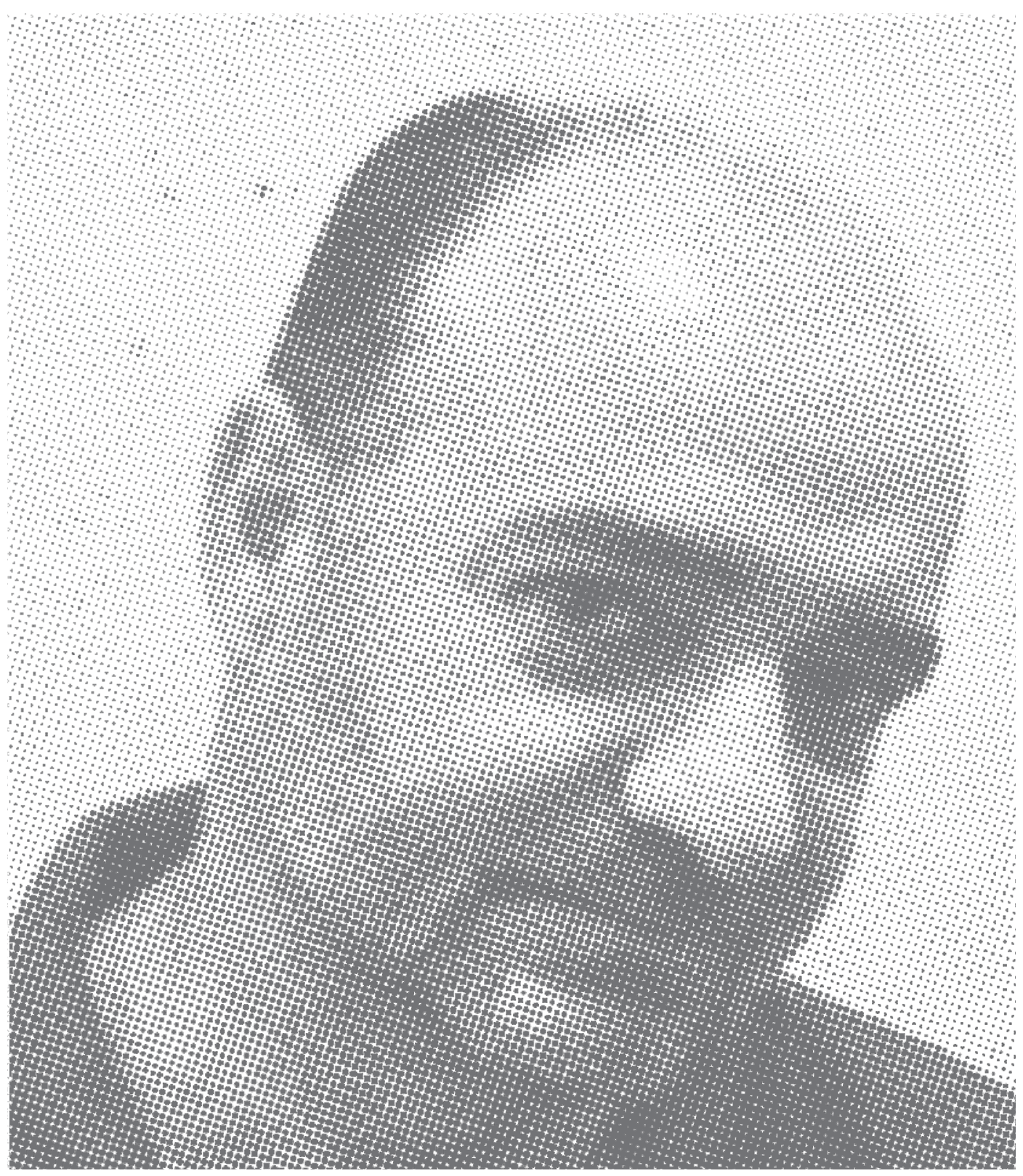




\section{Biónica: hacia la ciencia del diseño}

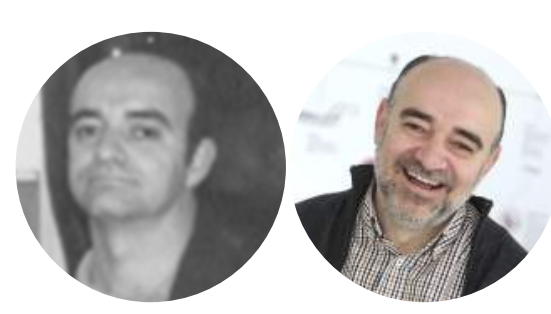

Gabriel Songel | gsongel@upv.es

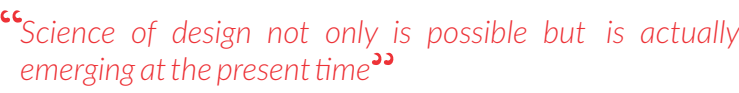

Herbert A. Simon

De Carmelo Di Bartolo, como de la mayoría de las personas con las que he trabajado y aprendido algo, solo puedo decir cosas buenas. Quisiera destacar algunas de sus facetas como Facilitador, Entusiasta, Motivador, Embaucador, Improvisador y un constante Incitador a la curiosidad. Todas ellas le convierten en un docente excepcional, que cualquiera que quiera dedicarse a la educación de cualquier materia, pero especialmente de diseño, debería cultivar todas aquellas facetas mencionadas. Conocí a Carmelo en una conferencia que dió en Alicante en 1985. Eran años de la expansión de la cultura española como el cine (Almodóvar), la música y el diseño.La conferencia de Alicante fue uno de los eventos más importantes en la España de los 80 por cuanto vinieron diseñadores internacionales de primer nivel como Alessandro Mendini o Philipe Starck, en pleno apogeo postmoderno. Aquel contexto era ideal para mi final de carrera de Bellas Artes en el que había pasado los dos últimos años especializándome en Diseño Industrial para optar a las becas de profesionalización que el gobierno de la Comunidad Valenciana había empezado a convocar.En el jurado de la selección para la beca estaba Carmelo y reparó en mis trabajos experimentales sobre la forma y en los proyectos de investigación que realizamos durante la carrera. El portfolio le importaba poco, ya que estaba buscando candidatos con capacidades investigadoras y experimentales para su incipiente Master en Diseño Biónico. Finalmente me seleccionó y aquello fue mi oportunidad para poder estudiar fuera de España.

El inicio del máster fue un descubrimiento de nuevos contextos personales, académicos y profesionales. La estancia en Milán y su entorno industrial fueron especialmente enriquecedores, y no solo por el desarrollo técnico que los productos y las empresas que los hacían demostraban tener. Fue especialmente motivador el pensar que todo aquello estaba por desarrollar en la España de aquella década. De hecho lo que encontré significativamente llamativo fueron las exposiciones de la Triennale, y en concreto la serie de Progetto Domestico. La capacidad discursiva, transversal e interdisciplinar sobre un mismo concepto y los montajes expositivos, es algo que hoy en día, treinta años después, tengo en mente cuando monto exposiciones de diseño e innovación.Siempre me llamó la atención la capacidad de Carmelo para convencer a los gobiernos autónomos de la Comunidad Valenciana y Castilla-La Mancha para financiar sus proyectos educativos, que luego se expandieron a Canarias, y toda América Latina. En 1993 tuve el privilegio de coordinar un número monográfico sobre Naturaleza, Diseño e Innovación en la publicación Temes de Disseny que editaba la Escuela de Diseño Elisava de Barcelona.Fue la ocasión de invitarle a participar y conocer a personajes del diseño como Gillo Dorfles, Werner Nachtigall, Ives Coineau y Biruta Kresling, Ezio Manzini, Jürgen Hennicke y Frei Otto. Aquella buena experiencia nos llevó a la preparación de un proyecto europeo para la catalogación de los proyectos fundamentados en la Naturaleza.

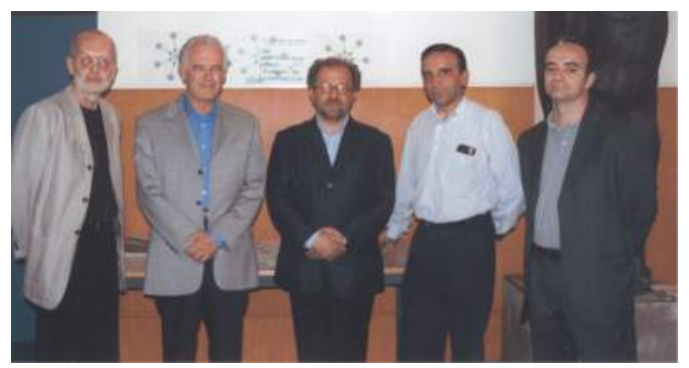

De izquierda a derecha: Jürgen Hennicke, Werner Nachtigall, Carmelo Di Bartolo, Carlos Plasencia y Gabriel Songel, en las reuniones preparativas del proyecto europeo en la UPV. 
La última colaboración que me pidió Carmelo fue en la publicación Experimenta sobre biónica. En concreto me centré en mi experiencia en el diseño de juegos de construcción y en el fundamento orgánico en muchos de esos juegos. Se me ocurrió llamarle "el fascinante juego de la Naturaleza". Aún lo sigo viendo y viviendo así por los innumerables productos que he podido desarrollar con esa premisa. Carmelo ha sido uno de esos facilitadores que me he ido encontrado en mi vida personal y profesional, como posteriormente han sido Ole Poulsen, vicepresidente de Lego, Manuel Lecuona, compañero y catedrático de Diseño en la UPV, Barney Lin, fundador de Gigo Toys en Taiwán, José Antonio Pastor, presidente de AEFJ, Asociación Española de Fabricantes de Juguetes o Peter Handstein, fundador de Hape Toys International. A todos ellos he tenido la ocasión de manifestarles mi agradecimiento. Ahora con este artículo, por si no lo hice en el pasado, quiero manifestarle nuevamente mi agradecimiento a Carmelo Di Bartolo.

\section{[LA NATURALEZA COMO ESTRATEGIA DE INNOVACIÓN]}

\section{[1. ARTE Y NATURALEZA]}

La naturaleza de la innovación, el arte de la innovación. El descubrimiento de la naturaleza como recurso de la innovación vino en mi carrera universitaria en Bellas Artes en Valencia (España). La búsqueda de estructuras abstractas y visualmente potentes me llevó al estudio del origen de la escritura [1] , símbolos y signos [2] , y a profundizar en el conocimiento de los artistas de vanguardia que habían tratado este campo desde diferentes ópticas desde Paul Klee a Malevich. Fue el estudio de Paul Klee [3] el que entró en la representación de estructuras naturales mínimas, estructuras de crecimiento, dibujos primitivos, dibujos infantiles. Klee fue capaz de dibujar en 1940 la imagen de un guerrero exactamente igual que un guerrero en una pintura primitiva descubierta en África en los 80 . Esta capacidad de síntesis de la imagen, de visualizar el pasado y de construir una teoría del arte moderno [4] al mismo tiempo, ha sido algo que aún me sigue inspirando, y especialmente me ha enseñado a seguir observando la Naturaleza desde diferentes ámbitos.

En 2018, en un paseo por la Sierra de la Calderona en Valencia encontré con una gran losa de 4 metros de larga con una triangulación en toda su superficie muy clara. Mientras analizamos la formación natural conocida como diaclasa, en la que se cuartea en dos direcciones una masa pétrea, cabría la posibilidad que fuese una intervención de la mano humana. La siguiente sorpresa fue encontrar construcciones gráficas primitivas con la misma composición. En septiembre de 2018 Henshilwood y Van Niekerk de la Universidad de Witwatersrand en Suráfrica, publicaron en Nature [5], la datación de más de 70.000 años de los dibujos de la Cueva Blosbom. Los dibujos son una triangulación entre tres líneas horizontales, una estructura visual muy parecida a la losa de Olocau. Esta coincidencia nos lleva a la reflexión de la indisoluble integración del ser humano con su entorno en tiempos ancestrales en contraste con la actual desintegración del entorno por el ser humano.
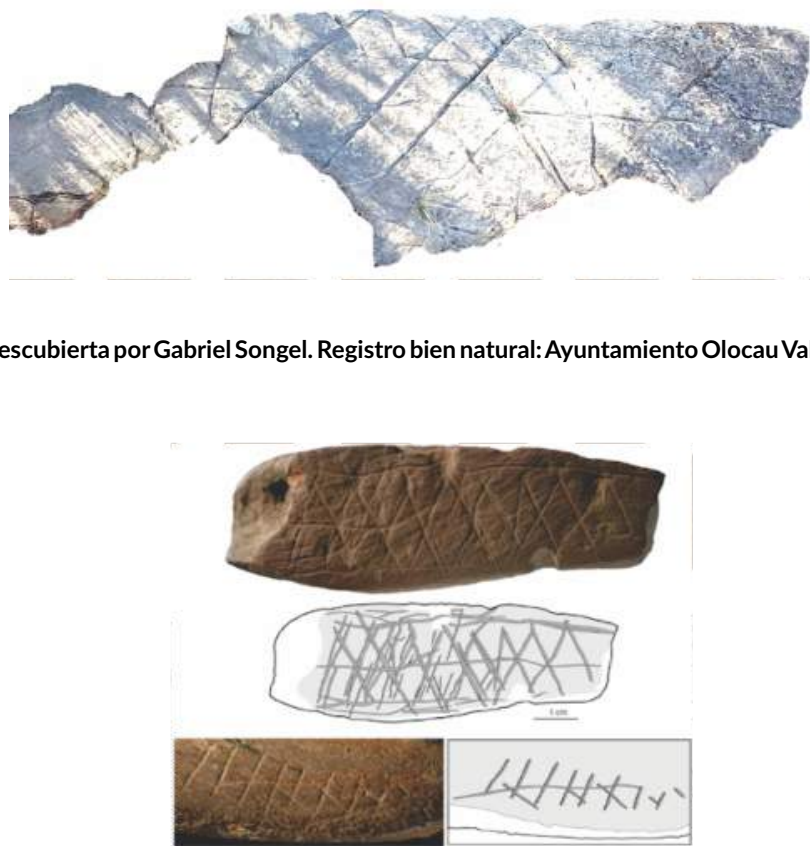


\section{[2. ESTRATEGIA CON MÉTODO PARA LA INNOVACIÓN]}

Con el desarrollo personal y profesional he llegado a la convicción que la innovación requiere de estrategia y método. Mi primera contribución en este sentido fue la tesis doctoral y el planteamiento metodológico. En segundo lugar, tuve el honor de coordinar un número monográfico de la revista Temes de Disseny publicada por la escuela Elisava de Barcelona en 1994 con el título Naturaleza Diseño e Innovación [6], en la que participaron autores de prestigio como Gillo Dorfles, Ezio Manzini, Yves Coineau y Biruta Kresling, Werner Nachtigall, Jürgen Hennicke o Carmelo di Bartolo. Mi contribución fue la de explicar el modelo metodológico de diseño basándome en el estudio de estructuras de la Naturaleza que había desarrollado en mi tesis doctoral.

\section{[NATURALEZA, DISEÑO E INNOVACIÓN: PROPUESTA METODOLÓGICA]}

Los modelos biológicos y el estudio de la naturaleza. El estudio de la naturaleza ha tenido y sigue teniendo múltiples y diferentes implicaciones en el diseño y la innovación. Como forma de analizar los sistemas y para el establecimiento de modelos biológicos, Bertalanffy, desde la aproximación científica de la teoría general de los sistemas, ya apuntaba a la biónica como factor de innovación.[7] Otros, como Pearce y Stevens, [8] han planteado la naturaleza como estrategia de diseño y otros como estrategia docente [9].

Es en estas líneas donde vamos a profundizar y plantear un modelo metodológico valedero en el campo didáctico, proyectual e investigador. De hecho, podríamos encontrar antecedentes del estudio de la naturaleza y su directa relación con el diseño básico en los cursos iniciales de la Bauhaus y posteriormente en la Escuela de Ulm. Estas experiencias han sido recogidas y puestas en práctica en la Sección de Diseño de la Facultad de Bellas Artes de la Universidad Politécnica de Valencia, de las que han derivado estudios metodológicos desarrollados en el Grupo de Investigación y Gestión del Diseño y su inclusión en las líneas de actuación de la investigación en diseño [10]. 4 Establecimiento de los niveles analógicos En el presente apartado vamos a analizar los diferentes procesos proyectuales o métodos utilizados hasta la actualidad, fijándonos especialmente en cómo ha sido el trasvase de información desde el análisis del sujeto natural a su aplicación práctica o proyectual. Si estudiamos todas las realizaciones que el hombre ha hecho tomando como referencia a la naturaleza, desde el mismo Leonardo hasta las últimas realizaciones del Centro de Investigaciones de Estructuras Naturales de Milán, podríamos agruparlas siguiendo el criterio de cuál ha sido la relación entre la referencia natural y su materialización en el mundo de lo artificial. Esta relación está medida de alguna forma por el grado de analogía, patente o no, entre la naturaleza y el objeto, entre el referente y lo referenciado, estableciendo como consecuencia cuatro niveles analógicos [11]:

1. Inconsciencia

2. Inspiración

3. Transposición

4. Imitación

Antes de entrar a definir cada uno de los niveles convendría tener en cuenta algunas consideraciones. En primer lugar se ha pretendido abarcar todas aquellas obras que de una forma u otra tienen alguna relación con la naturaleza para así contribuir al esclarecimiento o determinación de qué se considera, o podría considerarse, biónica y qué no. Esta consideración se desprende de la afirmación lógica de que no todo lo que haya tomado como referencia a la naturaleza ha de ser necesariamente visto como biónica. Tal sería el ejemplo de cualquier período artístico que haya asumido repertorios formales de la naturaleza. En segundo lugar, los niveles analógicos no se consideran ni como compartimentos estancos que clasifiquen las obras categóricamente, ni como vasos comunicantes en que todo se quede al mismo nivel. Habrá ejemplos en la historia del diseño que por sus condicionamientos particulares podrán enmarcarse entre un nivel y otro. Por último, y reafirmando la consideración anterior, habrá casos en los que, partiendo de una intención clara y preconcebida de una total imitación de un sujeto natural, por las sucesivas evaluaciones del proyecto y posibles inviabilidades de las propuestas, a la vista de los resultados, o en el mismo proceso proyectual, se vaya descendiendo a niveles anteriores. También puede ocurrir en sentido contrario, es decir, que, pretendiendo una simple inspiración, a medida que surjan los subproblemas se encuentren las soluciones en el sujeto natural de referencia, y por lo tanto el resultado pueda ser incluido en niveles posteriores. Con todo esto, el primer nivel, que denominamos de inconsciencia, agruparía a todas aquellas realizaciones que por métodos de diseño convencionales llegan, sin saberlo, a soluciones que se encuentran en la naturaleza. No queremos decir con ello que si se hubiera seguido un método biónico posiblemente se habría llegado antes a la solución, pues muchas de las resoluciones de la naturaleza son, constructivamente, elementales y con un proceso de pensamiento lógico se llegaría a soluciones similares. Éste es, de hecho, el objetivo del diseño básico.

Posiblemente el ejemplo más representativo, y anecdótico a la vez, de éste nivel sea el diseño de las cúpulas geodésicas de Buckminster Fuller de doble retícula espacial, cuyos resultados constructivos le 
resultaron "asombrosamente semejantes» a las diatomeas o a las geometrías de los cactus esféricos [12].De este nivel cabría añadir alguna cosa más, y es que contempla a un gran número de trabajos realizados hasta el presente, pero que no pueden considerarse enteramente dentro de la biónica, aunque los resultados sean muy similares a los aportados por la naturaleza, ya que no ha habido ni una intención previa, ni por lo tanto un método analógico específico para llegar a esas similitudes. Coineau y Kresling [13] incluirían en esta categoría a aquellos sistemas en los que las analogías han sido establecidas a posteriori, suponiendo los modelos naturales un recurso para el perfeccionamiento de las invenciones humanas. Un segundo nivel analógico sería el llamado de inspiración, por estar basado en una concepción parcial de la globalidad del sujeto natural referente que podría llevar al no respeto, o incluso a la contradicción, de los principios básicos que comporta esa globalidad. Es decir, este nivel estaría caracterizado por la toma anecdótica de algunos de los aspectos con que se manifiesta la naturaleza, sin tener en cuenta que éstos son consecuencia de un proceso evolutivo-funcional, y que están condicionados unos a otros, ya que, como sistema biológico, hay una interrelación entre el todo y las partes.

A este nivel pertenecerían todas aquellas obras que gratuitamente se han basado en formas biológicas $u$ orgánicas sin atender a las causas funcionales a que responden esas formas. No obstante, hay que reconocer que, tal y como afirma la psicología de la percepción, es precisamente por ese aspecto formal, sea razonado o no, por lo que puede empezar a reconocerse un objeto como basado en algo natural, y que, en caso de que fuese una forma razonada cercana a lo natural daría lugar a lo que podría llamarse una estética biónica. De esta forma, y tal como considerábamos anteriormente, los movimientos artísticos que exaltaban a la naturaleza como fuente de inspiración para la generación de formas tanto bidimensionales como tridimensionales no pueden considerarse dentro de la biónica. El tercer nivel analógico estaría caracterizado por la trasposición de los principios básicos observados en el sistema natural que se aplican sobre el objeto artificial y que, por lo general, definen el resultado. En este caso, a diferencia del anterior, la toma de aspectos "del referente puede ser parcial, pero en ningún momento entrará en contradicción con la armonía aglutinadora del mismo, o al menos esa trasposición parcial tendrá una justificación funcional. Por este motivo, a partir de este nivel se puede hablar de biónica propiamente dicha dentro de las definiciones ampliamente aceptadas: Es la ciencia de los sistemas, cuyo funcionamiento se basa en el de los sistemas naturales, o que presentan características específicas de sistemas naturales, o que tienen analogías con éstos (Steele, 1960) [14].8 La biónica no trata solamente de engineering, sino de encontrar ideas que permitan construir los más variados mecanismos cuyos prototipos vivientes existen desde hace siglos al alcance del hombre. Su proceso básico sería preguntarse cómo funciona, y posteriormente cómo reproducir este principio [15].

Es la utilización de prototipos biológicos en el diseño de sistemas sintéticos creados por el hombre. $\mathrm{O}$ sea, se trata de estudiar los principios fundamentales de la naturaleza y llegar a la aplicación de principios y procesos a las necesidades humanas [16]. Es el estudio de sistemas vivientes para aplicar a las tecnologías sus principios técnicos y procedimientos. Es particularmente apta para estimular la capacidad de captar los detalles tridimensionales y los principios formales que los estructuran, así como para incrementar la capacidad de transformación, es decir, cuando se examina y analiza un objeto análogo [17]. Bajo el término de biónica se estudian generalmente dos tipos de trabajos científicos: uno, más relacionado con el diseño, que estudia la naturaleza en su equilibrio entre formamateriales-funciones, tratando de encontrar soluciones utilizables por el hombre para su medio ambiente; el otro, que investiga principalmente los problemas neurofisiológicos de las formas vivientes con el objeto de reproducirlas artificialmente [18].

La finalidad de la biónica es descubrir los secretos de la naturaleza, sea en las plantas como en los animales, y deducir sus principios constructivos sobre los que basar las creaciones técnicas [19]. A este nivel pertenecerían los trabajos desarrollados por el Centro de Investigaciones de Estructuras Naturales del Istituto Europeo di Design de Milán y parte de las realizaciones del Instituto de Estructuras Ligeras de la Universidad de Stuttgart, ya que éste es el criterio seguido en sus métodos de trabajo según veremos en apartados posteriores. Para Coineau, un ejemplo de trasposición parcial sería el Palacio de Cristal de Paxton, en el que solamente el principio es tomado, y la realización técnica parece no tener nada que ver con su modelo viviente: la hoja circular flotante de la Victoria Amazónica, ya que es claramente muy diferente a las cristaleras rectangulares propuestas por el ingeniero [20]. Por último, la imitación total de la naturaleza sería el cuarto nivel analógico que se propone. Éste supondría la trasposición de todos los aspectos más importantes de un sujeto natural; por ejemplo, la función, estructura y forma, al sujeto artificial. Ejemplos de este nivel tendríamos a lo largo de la historia de la técnica, y más concretamente con las últimas investigaciones sobre el vuelo de los insectos, con vistas a su aplicación en hélices y turbinas, realizadas por Werner Nachtigall, del Instituto de Zoología de Saarbrücken. Sin embargo, Coineau no distingue diferencia entre este nivel y el anterior al afirmar: «En un buen número de casos, se trata de una copia de la naturaleza según un proceso biónico típico: estudio de un sistema natural, interpretación del principio y, después, la trasposición a una realización de tipo industrial»; y pone como ejemplos clásicos la piel artificial del delfín, que pertenecería al cuarto nivel, y las construcciones de Le Ricolais inspiradas en los 
radiolarios, que corresponderían al segundo o tercer nivel [21].

Posiblemente este nivel sea el más controvertido por diferentes razones que conviene considerar. Por una parte, la intención de imitar a la naturaleza con el convencimiento primero de que era posible, y segundo de que aportaba soluciones óptimas, ha quedado demostrado por la historia, desde Leonardo hasta la actualidad, que no siempre es factible o exitosa. Esta afirmación puede quedar ilustrada con la historia de la aviación, en la que el hombre ha podido volar cuando se ha olvidado de pretender elevarse por principios mecánicos, tal y como pensaba Leonardo, y ha experimentado a partir del vuelo a planeo. Por otra parte, el conocimiento progresivo de los sistemas neurofisiológicos de los seres vivos y el desarrollo paralelo de la microelectrónica y la tecnología permiten multitud de puntos de encuentro entre unas ciencias y otras, adoptando los nombres de cibernética, rebotica o biomecánica; siendo, por lo tanto, los límites entre unos y otros perfectamente permeables.

Quizá por esta razón, este nivel sea el que se preste a una dualidad de reconocimiento; por un lado como el más asimilable con el término de biónica, ya que se da una equivalencia mayor entre el referente natural y lo artificial; y, por otro lado, como puerta abierta a otras áreas del conocimiento científico que pueden llevar a la confusión. Con el establecimiento de los niveles analógicos, tenemos a nuestra disposición una herramienta de medida y de acceso a los modelos metodológicos que comporta la biónica.

\section{[3. La matriz de la creatividad: una hoja de ruta para una trayectoria profesional y de investigación en diseño]}

Con esta matriz se pretendía hacer ver todo un panorama de campos en los que se podían aplicar principios de diseño con fundamentos en la Naturaleza [22] , de forma que fuera lo más sugerente posible para los diseñadores e investigadores en este campo. Efectivamente, este esquema, con el paso del tiempo, has sido una hoja de ruta que se ha ido formando a medida que han ido surgiendo las oportunidades para aplicar una metodología biónica. 25 años después compruebo que sigue siendo sugerente porque aparecen nuevos proyectos en los que se puede seguir aplicando o generar nuevas ideas.

\begin{tabular}{|c|c|c|c|c|}
\hline \multirow[t]{2}{*}{ SECTORES } & \multicolumn{4}{|l|}{ ARGUMENTOS } \\
\hline & $\begin{array}{l}\text { Construcciones } \\
\text { ligeras }\end{array}$ & Articulaciones & Locomoción & Superficies estructuradas \\
\hline Mobiliario & $\begin{array}{l}\text { Muebles ligeros } \\
\text { Estructuras } \\
\text { Estanterias }\end{array}$ & $\begin{array}{l}\text { Elementos } \\
\text { de cerrajeria: } \\
\text { Bisagras } \\
\text { Manillas } \\
\text { Tirantes }\end{array}$ & Rodamientos & $\begin{array}{l}\text { Rigidez de materiales } \\
\text { laminados }\end{array}$ \\
\hline Juguetes & $\begin{array}{l}\text { Juegos constructivos } \\
\text { Arquitecturas }\end{array}$ & $\begin{array}{l}\text { Móviles } \\
\text { Muñecos }\end{array}$ & $\begin{array}{l}\text { Juguetes mecánicos } \\
\text { Robots }\end{array}$ & $\begin{array}{l}\text { Juegos táctiles } \\
\text { Puzzles }\end{array}$ \\
\hline Sistemas constructivos & $\begin{array}{l}\text { Sistemas estructurales: } \\
\text { Stands } \\
\text { Andamios }\end{array}$ & $\begin{array}{l}\text { Uniones } \\
\text { Rotulas } \\
\text { Juntas }\end{array}$ & $\begin{array}{l}\text { Ultraligeros } \\
\text { Deltas } \\
\text { Chasis } \\
\text { Carrocerias }\end{array}$ & $\begin{array}{l}\text { Planeles } \\
\text { Perfiles } \\
\text { Cubiertas }\end{array}$ \\
\hline Embalaje & $\begin{array}{l}\text { Envases minimos } \\
\text { Mallas } \\
\text { Bolsas } \\
\text { Composites }\end{array}$ & $\begin{array}{l}\text { Plegados } \\
\text { Enganches } \\
\text { Broches }\end{array}$ & $\begin{array}{l}\text { Sistemas de transporte } \\
\text { de seguridad }\end{array}$ & $\begin{array}{l}\text { Rigidez de materiales } \\
\text { pobres: cartón, papel, } \\
\text { plásticos }\end{array}$ \\
\hline $\begin{array}{l}\text { Materiales } \\
\text { de construcción }\end{array}$ & $\begin{array}{l}\text { Ladrillos } \\
\text { Celosias } \\
\text { Forjados } \\
\text { Mallas }\end{array}$ & $\begin{array}{l}\text { Uniones } \\
\text { Colágenos }\end{array}$ & $\begin{array}{l}\text { Materiales ligeros } \\
\text { y de alta resistencia }\end{array}$ & $\begin{array}{l}\text { Tejas } \\
\text { Pavimentos } \\
\text { Revestimientos }\end{array}$ \\
\hline Textil & Fibras ligeras & $\begin{array}{l}\text { Enganches } \\
\text { Cierres } \\
\text { Botones } \\
\text { Broches }\end{array}$ & Tejidos transpirables & $\begin{array}{l}\text { Mallas articuladas } \\
\text { Composites } \\
\text { Caracterización suave, } \\
\text { áspero, blando }\end{array}$ \\
\hline
\end{tabular}

\section{[4. PROYECTO LEGO ZNAP]}

Una de estas oportunidades únicas en la vida, apareció con Lego, multinacional danesa de juegos de construcción. El gran reto permanente de los juegos de estructuras ha sido el conseguir niveles de libertad de construcción como las estructuras de alambre hechas por niños africanos mencionadas anteriormente. Otra de las desventajas de los juegos de estructuras tradicionales es que son construcciones estáticas, es decir, que para variar la forma hay que desmontar lo ya realizado. 


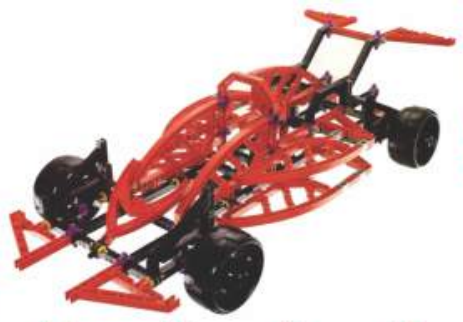

De todos estos problemas Lego fue consciente y con esta motivación desarrolló el ZNAP system, lanzado en el Reino Unido y Alemania entre 1998 y 2000 . Lego es conocida por los juegos de ladrillos de plástico como juego de construcción, pero no como juego de estructuras. La principal diferencia entre un tipo de juegos y otros es que, mientras que un juego de construcción las piezas pueden ponerse unas encima de otras sin conexión, en los juegos estructurales, es la geometría que configura unión lo que condiciona el volumen final. Esto es, mientras que todos los juegos estructurales son juegos de construcción, no todos estos se pueden considerar estructurales.

Pero ¿cuál es el problema de los juegos de estructuras? Que si el resultado del juego son formas abstractas, los niños se aburren inmediatamente. La geometría del nudo limita la creatividad del niño, ya que, en algunos sistemas, es realmente difícil dar con el ángulo correctamente. Por otra parte, suelen ser sistemas estáticos, imposibles de transformar. Hay que desmontarlos para construir otra cosa diferente. Lego era muy consciente de estos problemas y de los límites inherentes a los juegos tanto estructurales como de construcción, de los cuales hay más de cincuenta tipologías en el mercado. El conocimiento como valor estratégico. Con el planteamiento de la investigación de un nuevo sistema estructural, la propuesta desde el Grupo de Investigación y Gestión del Diseño fue la de seguir el modelo metodológico planteado en la tesis doctoral.

\section{[ETAPA 1. PLANTEAMIENTO Y ANÁLISIS DE LAS NECESIDADES]}

Se presentará la necesidad en forma de enunciado lo suficientemente genérico como para ser trasladado a un argumento biónico. Debe demostrarse una necesidad económica y una disponibilidad de satisfacerla.

\section{[K'NEX]}
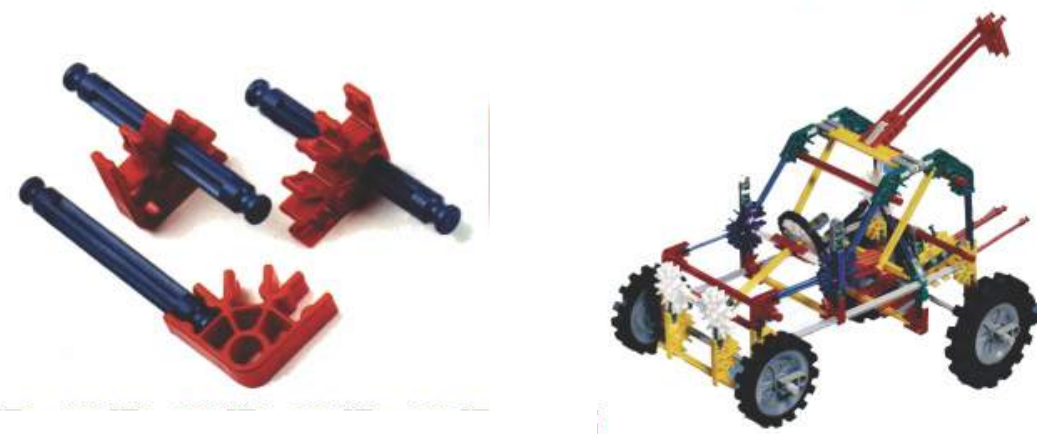

En el caso de Lego, la necesidad era diseñar un sistema estructural que fuese competitivo con los aparecidos contemporáneamente en el mercado como K'nex de Hasbro, y Construx de Mattel. Un sistema que fuese transformable.

\section{[ETAPA 2. IDENTIFICACIÓN DEL PROBLEMA]}

Con los datos precedentes y con la información técnica, se identifica el problema y se establece el argumento biónico que en el mundo natural puede presentar soluciones a ese mismo problema. El planteamiento del argumento biónico tendrá un equilibrio entre lo genérico y lo específico, permitiendo centrar el tema a investigar pero sin llegar a dar o sugerir soluciones concretas. El proyecto se identificó con el argumento biónico "articulaciones", por ser el campo conceptual menos tratado en los juegos estructurales, ya que la mayoría son sistemas fijos, como hemos dicho anteriormente, y por lo tanto donde mayor innovación se podía alcanzar.

\section{[ETAPA 3. CONCEPTO DEL PROYECTO]}

Consiste en la búsqueda de posibles soluciones manifestadas en la Naturaleza. Es una etapa que requiere capacidad de sintetizar el enunciado, capacidad de observación y reconocimiento de ese enunciado en diferentes realidades del mundo natural. En definitiva, capacidad analógica, que puede 
suplirse en algunos casos por la disposición de mucha información visual de diferentes ámbitos de la Naturaleza. Se elegirán aquellos sujetos naturales que mejor representen al argumento enunciado. Conduce a más de una concepción del proyecto. Las soluciones que sirvieron como referencia para el estudio fueron los diferentes sistemas de rótulas, las articulaciones en los esqueletos y, especialmente, las articulaciones rígidas de los crustáceos.

\section{[RÓTULAS, ESQUELETOS, CRUSTÁCEOS, ARTICULACIONES RÍGIDAS]}
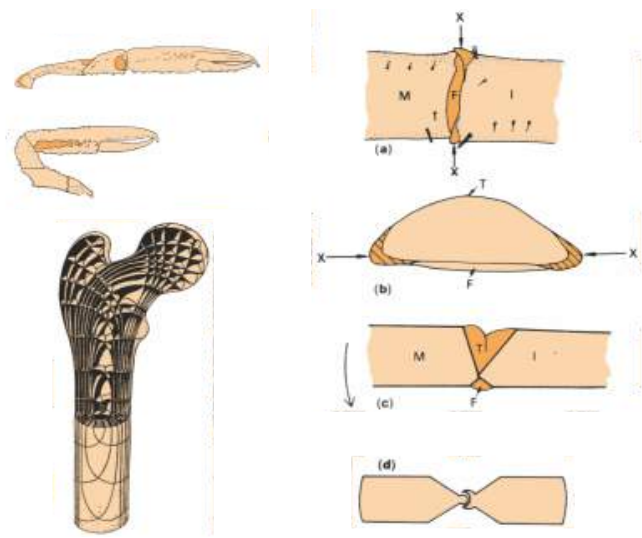

[ETAPA 4. ANÁLISIS SUJETOS NATURALES]

Se analiza cada uno de los sujetos naturales seleccionados en la etapa anterior. El análisis específico constará de:

- Diferenciación de los mecanismos del sujeto natural

- Estudio de las relaciones formales entre ellos

- Comprensión de la naturaleza y organización de los materiales

- Estudio de la estructura funcional

Se preservará la información a través de fotografías, gráficos, esquemas y maquetas que sinteticen las propuestas formales observadas. Todos los principios constructivos de las articulaciones analizadas fueron dibujados teniendo en cuenta el principio constructivo de las piezas de Lego.

\section{[ETAPA 5. PROPUESTAS DE APLICACIÓN]}

De los análisis anteriores se irá realizando una exhaustiva relación de posibles aplicaciones, sin descartar aquellas que parezcan disparatadas, utópicas o inalcanzables. Aquellas ideas que aportaban una mayor versatilidad al sistema, y que empezaban a satisfacer el objetivo del proyecto, fueron experimentadas con algunas de las más de dos mil piezas que lego ya tiene en su catálogo.

\section{[ETAPA 6. ESTUDIOS DE MERCADO Y VIABILIDAD ECONÓMICA]}

Se procederá al estudio de lo existente en el mercado tanto en lo referente al problema proyectual, como en lo concerniente a las manifestaciones del argumento biónico en productos ya realizados. Se realizará una exhaustiva relación de posibles áreas de intervención bien en cuanto a nuevos productos, o bien en cuanto a mejoras competitivas con lo existente. Tras la comparación entre esta relación y la obtenida en la etapa anterior se podrán detectar las áreas de interés, o incluso, productos concretos a desarrollar. Una doble investigación de mercado se realizó paralelamente. Por un lado se analizaron los sistemas estructurales de los competidores, detectando puntos fuertes y puntos fuertes, y por otro lado se estudiaron los nudos estructurales que existen en el campo de la construcción civil para andamios, stands de ferias, etc. analizaron los sistemas estructurales de los competidores, detectando puntos débiles y

[MECCANO]
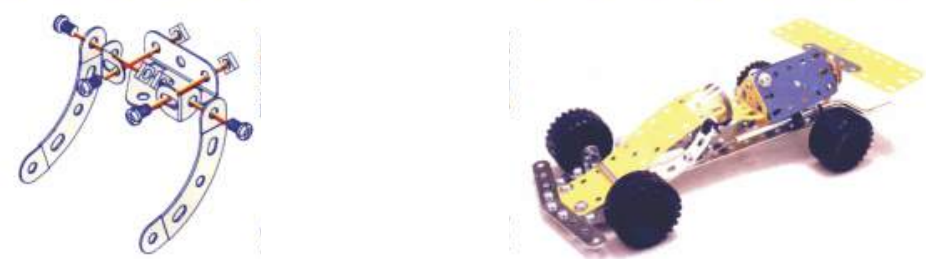


\section{[CONSTRUX]}

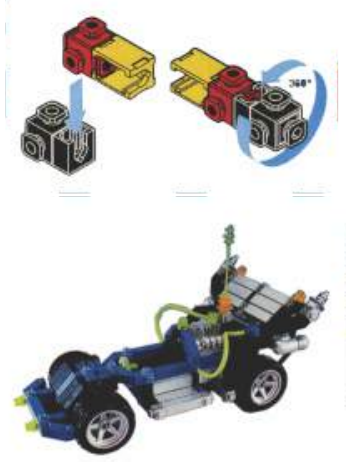

[TRIDI 2000]

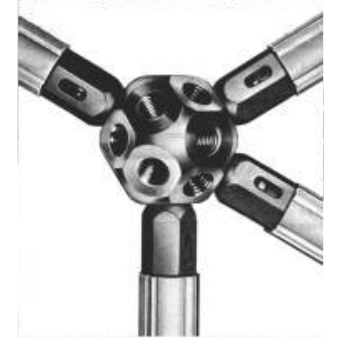

[STEWARTS \& LLOIDS]

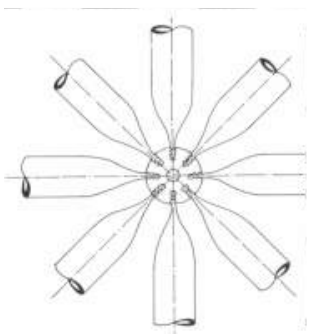

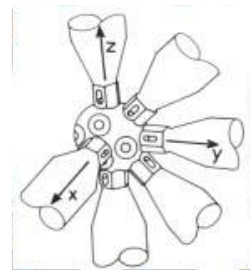
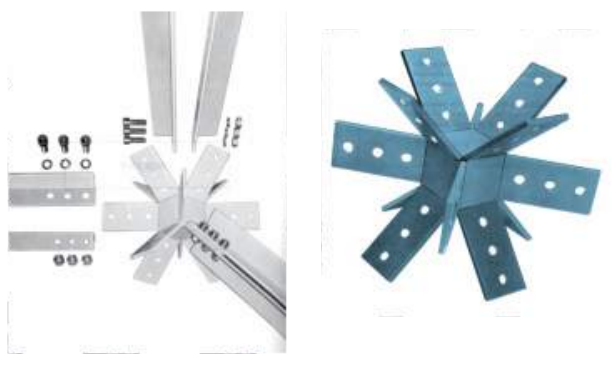

[SISTEMA MAS]
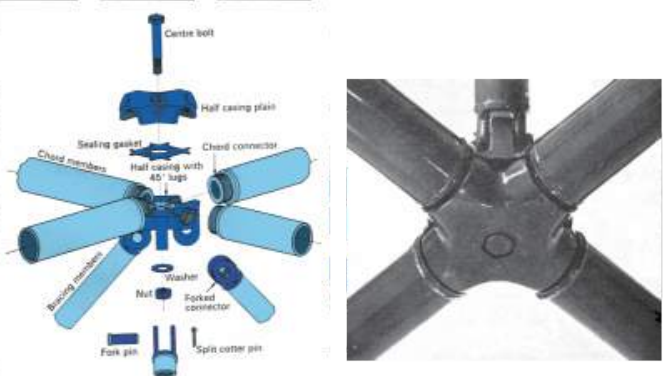

[SISTEMA WACHSMANN]

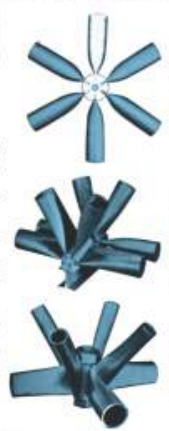

Evidentemente en la investigación del concepto del nuevo sistema, en la que ha participado el autor, se pretendió experimentar las metodologías descritas en el método. Era lógico, por tanto si pretendíamos obtener un sistema flexible y transformable, tener como referente la naturaleza no solo para obtener conceptos constructivos, sino para provocar la fascinación por la geometría y la intuición hacia la experiencia constructiva. Con estas premisas se estudiaron sistemas de articulación de elementos rígidos como las pinzas de los crustáceos o las microrótulas de las púas de los erizos y así se obtuvieron los principios de giro de las piezas y el concepto de construcción o crecimiento del nudo según las necesidades estructurales.

En ambas investigaciones se detectó un hueco en el mercado el concepto innovador de un sistema estructural transformable. Al coincidir este hueco detectado con las propuestas de aplicación provenientes del análisis biónico, se cerraba el proceso de investigación para la obtención de algún concepto innovador. El desarrollo de la idea en el que intervinieron diseñadores industriales propios de la compañía, llevó a definir el sistema Lego ZNAP, que fue lanzado a finales de 1998 en el norte de Europa. 


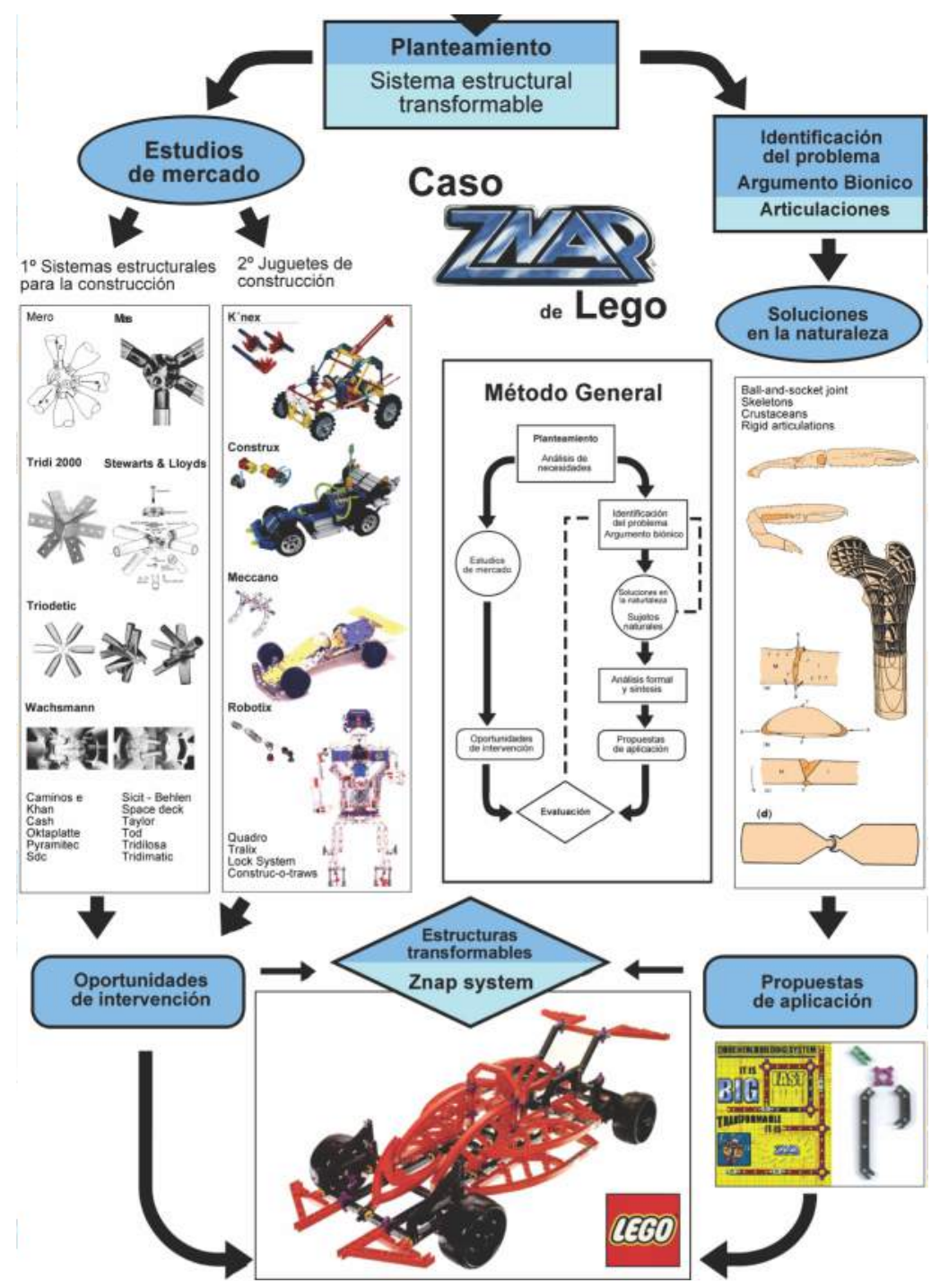

El sistema está compuesto por 20 elementos estructurales incluyendo dos nudos y un codo articulado. La innovación del sistema está en la forma de unir los nudos con las traviesas que permite dos funciones: girar la traviesa sobre el nudo sin desmontarlo, y generar la tercera dimensión atravesando un nudo sobre otro. La primera función da la posibilidad de fijar la traviesa en cuatro posiciones diferentes y la segunda permite añadir una pieza con dos salidas (eje $\mathrm{x}$ ) con otra de cuatro salidas (ejes y,z). De esta manera el niño puede construir el nudo dependiendo de sus necesidades y sin tener conocimientos previos de geometría. Cuando necesita más conexiones solo tiene que añadir un elemento al nudo y no necesariamente tiene que desmontarlo todo para volverlo a construir. Esta capacidad de giro y de crecimiento convierte al sistema Lego Znap en el primer juego de estructuras transformables donde, a la diversión tradicional de construir, se añade el descubrimiento de la geometría a través de la experiencia transformadora.
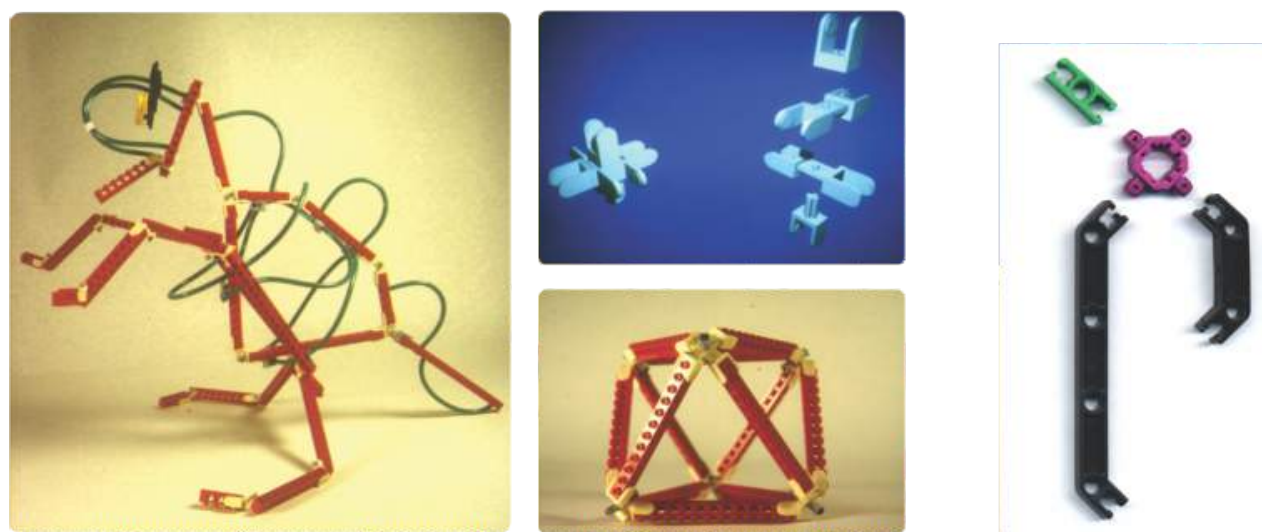


\section{[5. ESTÉTICA ORGÁNICA EN PARQUES INFANTILES]}

En aquel proyecto se proponía definir y revisar las características de los parques infantiles para llegar a determinar requisitos y propuestas concretas de elementos de juego, consecuentes con el principio de accesibilidad e integración social. Hasta entonces los parques infantiles públicos eran estructuras arquitectónicas construidas con postes y paneles por cuestiones puramente de facilidad de fabricación y montaje. Sin embargo todos los fabricantes recurrían a los mismos sistemas constructivos olvidándose del requerimiento de la inclusividad en el juego para los usuarios con disfunciones motoras. [23]

Fue un proyecto financiado con fondos FEDER y el Ministerio de Industria de España, realizado con AIJU Instituto Tecnológico del juguete e industrias afines y la empresa Parques Infantiles ISABA. A partir de las investigaciones generadas en el proyecto, se exploran nuevos conceptos de diseño de productos desde de las limitaciones de los usuarios con problemas de movilidad. De este modo, los parques pueden ser utilizados por todos los niños y pierden la apariencia ortopédica que utilizan la mayoría de empresas especializadas. Fue determinante la estética orgánica que caracterizó a toda la línea como algo realmente diferenciador de lo existente en el mercado y sugerente para la interacción con niños y niñas con o sin discapacidad. La propuesta de diseño cumple las normativas a nivel europeo y americano y se presenta al público en dos formatos: como línea de diez productos, y como parque tematizado.La primera aplicación fue la construcción de catorce elementos de juego en el parque temático de Terra Natura en Benidorm. La segunda aplicación fue el diseño y desarrollo de la línea de productos compuesta por diez elementos de juego con el nombre de ReCrea producida por ISABA que ha ido saliendo al mercado paulatinamente en este periodo, hasta ser presentado en ferias especializadas en mobiliario urbano y premiado por su valores de integración a niños discapacitados.

El conocimiento generado y la experiencia desarrollada fue reconocida por AENOR al invitarme a participar en el comité de normalización nacional y la representación de España en el comité europeo y difundida a través de la última normativa publicada en 2009.La importancia de la realización de los prototipos reales, es la materialización de la obra del diseñador, que culmina en la presentación de una feria especializada. La implantación del producto en las ciudades es la culminación de la función social del diseño y la innovación que supone la accesibilidad para todo tipo de usuario infantil. La construcción real del proyecto fue desarrollada años más tarde con la instalación del "Children's Temple" en Terra Natura por la empresa ParcNatur. Se trata del diseño y construcción de catorce elementos de juego en el parque temático de Terra Natura en Benidorm aplicando los principios de accesibilidad para todos los niños con o sin discapacidad motora. El resultado fueron estructuras tematizadas con laberintos, paneles de juegos, mallas colgantes, hamacas balancines, rampas y toboganes totalmente accesibles.
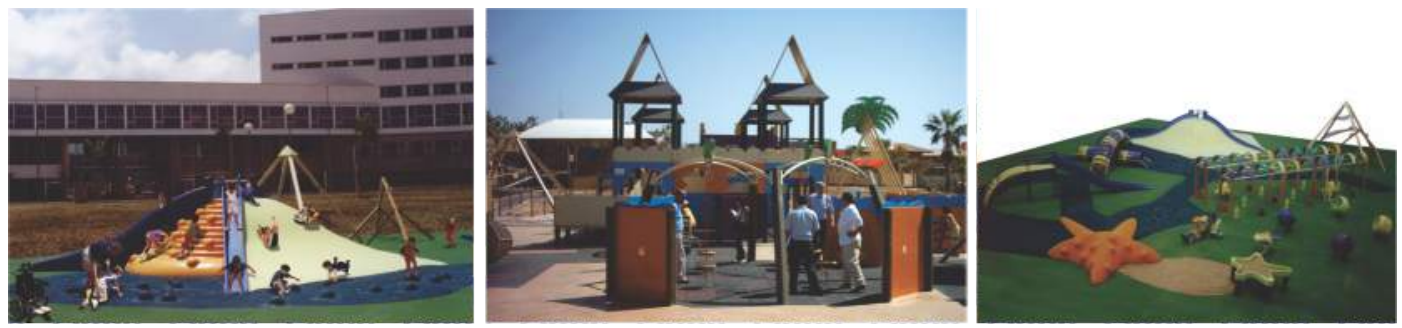

El portal Hosteltur, Comunicación para el turismo del futuro, avalada por la Sociedad Estatal para la Gestión de la Innovación y las Tecnologías Turísticas, S.A (SEGITTUR) presentaba la apertura del parque con estas características de accesibilidad como de las primeras de Europa. Así lo referenciaba también el diario El Mundo, en la sección "Hacia la igualdad" patrocinada por la ONCE en su edición digital,"Terra Natura tendrá el primer parque adaptado a niños discapacitados de Europa". El portal de información turística en inglés www.benidorm-spotlight.com lo presenta como un parque infantil accesible e innovador.

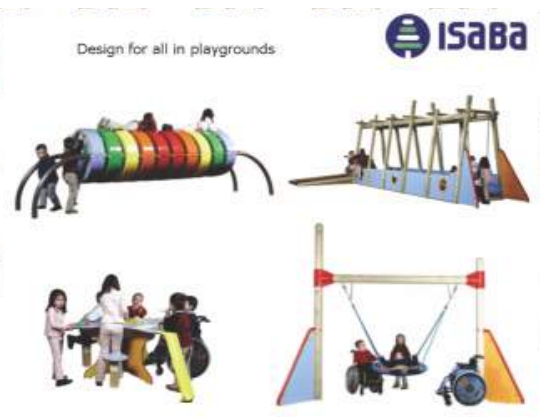


El desarrollo de la línea de productos fue la segunda aplicación de la investigación, con el diseño y desarrollo de la línea de productos compuesta por diez elementos de juego con el nombre de ReCrea producida por la empresa ISABA que ha ido saliendo al mercado paulatinamente en este periodo, hasta ser presentado en ferias especializadas en mobiliario urbano y premiado por su valores de integración a niños discapacitados. La línea ReCrea fue reseñada en varios medios especializados dada su novedad estética e innovación constructiva. Esta línea de productos ha sido presentada en ferias profesionales como TEM TECMA Feria Internacional del Urbanismo y del Medio Ambiente en Madrid 2006. En 2009 la línea ReCrea fue premiada por la Design for all Foundation, organización internacional de prestigio en el campo del diseño y la arquitectura dedicada a la difusión de valores de integración social y accesibilidad para todos los públicos.

\section{[6. PROYECTO ENDOSCOPIA]}

El proyecto es la aplicación práctica de las metodologías del diseño biónico planteadas en la tesis doctoral del año 1991. Es un ejemplo perfecto de la tradición científica de recurrir a diseñadores para la visualización y construcción de nuevos sistemas médicos. El proyecto de investigación recibió la financiación del Instituto de Salud Carlos III y consistió en la realización de un prototipo que permitiera la exploración del aparato digestivo. Se trata de un endoscopio de doble balón que dispone de un sistema de traslación automatizado basado en cavidades hinchables. El dispositivo comprende medios de traslación y medios electrónicos que controlan la actuación de éstos. Entre los medios de control figura una interfaz hombre-máquina.La búsqueda de referentes en la naturaleza que realizan el mismo tipo de desplazamientos como las lombrices, las anémonas marinas, hidras, medusas o miriápodos.

\section{[RECOPILACIÓN DE INFORMACIÓN DEL SISTEMA DE LOCOMOCIÓN EN ENDOSCOPIAS]}

\section{[ANTECEDENTES]}

Con la endoscopia convencional se puede visualizar el esófago, el estómago, el duodeno y con habilidad, los primeros $\mathrm{cm}$. del yeyuno. Con la colonoscopia convencional se puede explorar de forma retrógrada todo el intestino grueso y con destreza los últimos $\mathrm{cm}$ del intestino delgado (ileon terminal) en el $85-90 \%$ de los casos. Ambas técnicas permiten además de observar las distintas estructuras anatómicas y objetivar la existencia de lesiones, tomar muestras para estudio anatomopatológico y actuar terapéuticamente cuando la situación lo requiere. El resto de tracto gastrointestinal, unos 7 metros de intestino delgado solo puede alcanzarse mediante métodos directos e indirectos, no exentos de limitaciones e incomodidad tanto para el explorador como para el paciente.

De los últimos avances tecnológicos encontramos sistemas robóticos para medicina intestinal podemos mencionar la más avanzada llamada peristáltica basada en un sistema de doble globo que permite replegar el intestino, los "gusanos viajeros" por control remoto, y las "píldoras navegadoras". La contribución desde el Grupo IGD Investigación y Gestión del Diseño de la UPV, fue la búsqueda y análisis de referentes en la naturaleza. Se trataba de identificar sistemas de locomoción de organismos en la naturaleza que realicen desplazamientos similares a los requeridos por el sistema de endoscopia. Se contrastó la información de los sistemas naturales y artificiales para identificar las propuestas que permitan ser trasladadas al proyecto.

\section{[INTRODUCCIÓN]}

Un objetivo de este apartado era interpretar la estructura de aquellas plantas y animales de los que tenemos cierta información mecánica en función de los principios de la ingeniería mecánica. Los sistemas mecánicos de sostén de los organismos multicelulares son complejos en cuanto a que cada uno consta de más de un tipo de elemento estructural, y cada tipo realiza una función diferente. El diseño, tanto del elemento individual como del sistema considerado como un todo, es obviamente más complejo que el diseño de una estructura de ingeniería, donde la misión de cada elemento está definida con mucha precisión.

Según Wainwright [24] , varios son los referentes a tener en cuenta:

1. Cilindros con fibras enrolladas como sistemas de membrana reforzados. Es el tipo de sostén neumático o hidrostático, en donde un volumen central de fluido sometido a presión está rodeado de una cubierta cuyo material está bajo tracción. En estos casos, las paredes celulares y corporales de los organismos sometidos a presión se refuerzan de un modo similar por fibras orientadas helicoidalmente. Un cilindro abierto cuya pared contenga fibras inextensibles en disposición helicoidal cruzada, puede presentar distintas formas que varían desde formas muy largas y delgadas con un ángulo de fibra pequeño hasta formas cortas y gruesas con un gran ángulo de fibra. 


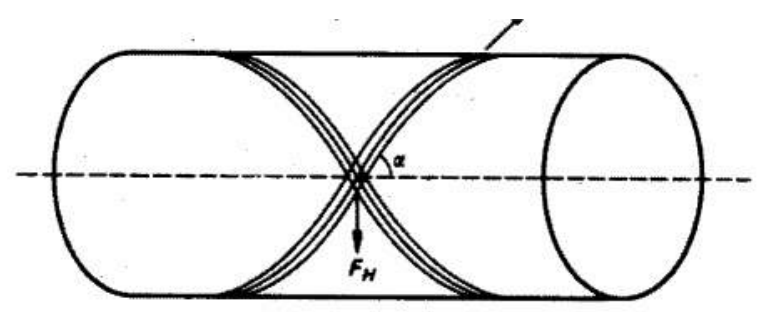

\section{[2. SISTEMAS FLUIDOS DE SOSTÉN EN PLANTAS Y ANIMALES: GUSANOS ]}

En todos los sistemas de sostén de los organismos hay dos modelos compresivos: el uno basado en material fluido y el otro en material sólido. Los organismos cuya resistencia a la compresión es fluida, son cilíndricos o sub cilíndricos. Lo que tienen en común todos los sistemas fluidos es un volumen de fluido central sometido a presión. La presión es producida por las fuerzas musculares de tracción en los animales y por fuerzas compresivas de turgencia en las plantas. Los gusanos tienden a tener el cuerpo más cilíndrico, la sección redonda y poseen gruesas cutículas formadas de muchas capas que contienen colágeno fibrilar. Las fibras son paralelas entre sí y envuelven helicoidalmente al animal en disposición fibrilar cruzada.

\section{[3. SISTEMAS TURBELARIOS: SEPIAS Y CARACOLES]}

Son los organismos que se mueven arrastrándose en un rastro de mucus sobre la superficie ventral, aplastada y ancha, o bien por deslizamientos musculares que dependen del aplastamiento dorsoventral característico de estos organismos. Poseen una membrana basal a la que están unidas los músculos longitudinales y disponiéndose transversalmente alrededor del cuerpo del organismo.

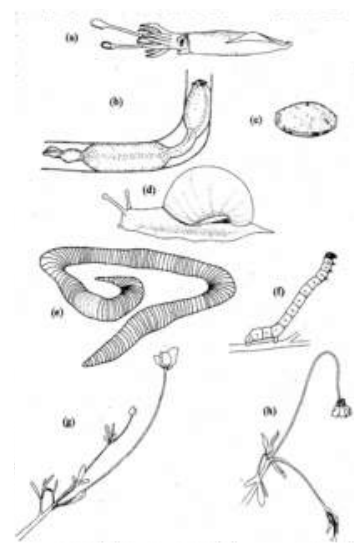

\section{[4. CILINDROS EXTENSIBLES Y ABIERTOS: ANÉMONAS DE MAR]}

Este modelo es, en esencia, un cilindro abierto cuya pared es tan flexible como para colapsar incluso bajo su propio peso. Las cavidades intestinales de las anémonas de mar permiten, mediante movimientos controlados de abrir y cerrar la boca ingiriendo o expulsando agua de mar, una enorme gama de formas y tamaños corporales. Las anémonas pueden abrir su boca, contraer sus músculos y contraerse hasta adquirir la forma de una masa arrugada, o bien puede ingerir agua de mar y dilatarse formándose una columna.

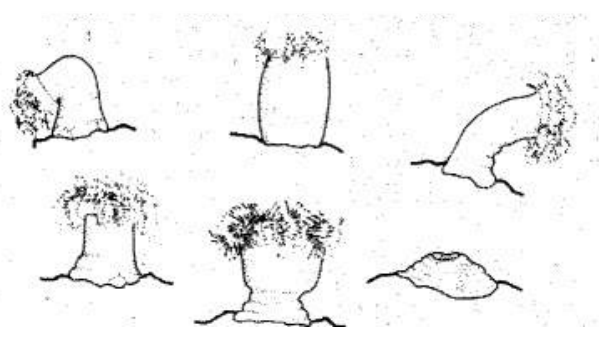

\section{[5. HIDRA]}

Carecen de estructuras internas pero su masa llamada mesoglea está poblada de fibrillas de colágeno orientadas paralelas en áreas de disposición ortogonal como fibras de refuerzo. Una de las 
propiedades mecánicas de la mesoglea es que, una vez alargada vuelve instantáneamente a su longitud de reposo cuando se quita la fuerza deformadora. Esta recuperación instantánea de la deformación de largo alcance es una propiedad desconocida a señalar en los materiales cuya composición es preferentemente colágeno, pero indica que la hidra, así como las anémonas, tienen una forma y tamaño de equilibrio que puede mantenerse sin necesidad de trabajo muscular o ciliar.

\section{[6. MEDUSAS]}

Las medusas nadan por contracciones pulsátiles de músculos circulares y a veces radiales situados sobre la superficie inferior de la umbrela, a una velocidad aproximada de una pulsación por segundo. La contracción muscular flexiona la umbrela, estirándose la superficie externa y comprimiéndose el volumen del material de la campana. Algunas medusas durante cada impulso motriz el grosor de la mesoglea experimentaba un aumento del 16 al 34 por 100 coincidente con una disminución del 56 por 100 de la superficie interna de la umbrela.

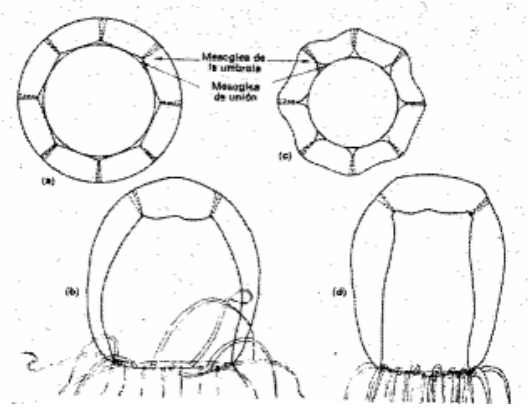

\section{[7. ESTRUCTURAS ARTICULADAS DE MATERIALES SÓLIDOS: MIRIÁPODOS CORREDORES Y MINADORES]}

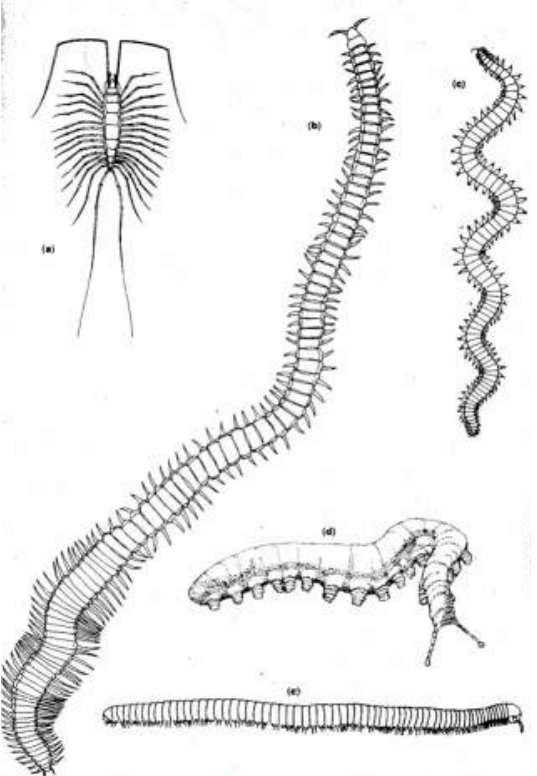

En los miriápodos, los movimientos enérgicos son producidos por músculos cuyas secciones transversales tienen gran superficie. La locomoción a alta velocidad se lleva a cabo por pasos más largos y más rápidos. Un miembro largo que posea músculos fuertes y gruesos será más pesado y difícil de mover con rapidez: los músculos largos y delgados pueden producir pasos más rápidos pero menos potentes. Un músculo largo puede desplazar su inserción con mayor amplitud o mayor ángulo que uno corto, y ésta es otra característica del diseño de las criaturas corredoras de largas patas: cada paso puede ser bastante largo en relación a la longitud del cuerpo. Los artrópodos más conocidos son los ciempiés.

El principio observado que la locomoción rápida y ágil exige más unidades neuro-musculares, requería una complejidad mayor de procesos coordinadores que la locomoción lenta pero potente.

Planteamiento de nuevos modelos de locomoción. Del análisis de la información obtenida en los apartados anteriores obtuvimos una serie de principios de diseño que se van a aplicar en los primeros modelos. La

visualización de los nuevos mecanismos de desplazamiento se realizó primero por ordenador. Posteriormente se valoró el sistema óptimo de acuerdo con los requerimientos del sistema médico y se construyeron los prototipos.

Seguidamente, aplicando principios de diseño industrial, se reprodujeron con materiales artificiales los mecanismos que mejor se adaptaban a las necesidades planteadas por el equipo de los médicos especializados en endoscopias.

El proyecto interdisciplinar contó con la participación conjunta de diseñadores, ingenieros y médicos procedentes de la Universidad Politécnica de Valencia y de la Fundación para la Investigación Hospital la Fe (Valencia). 

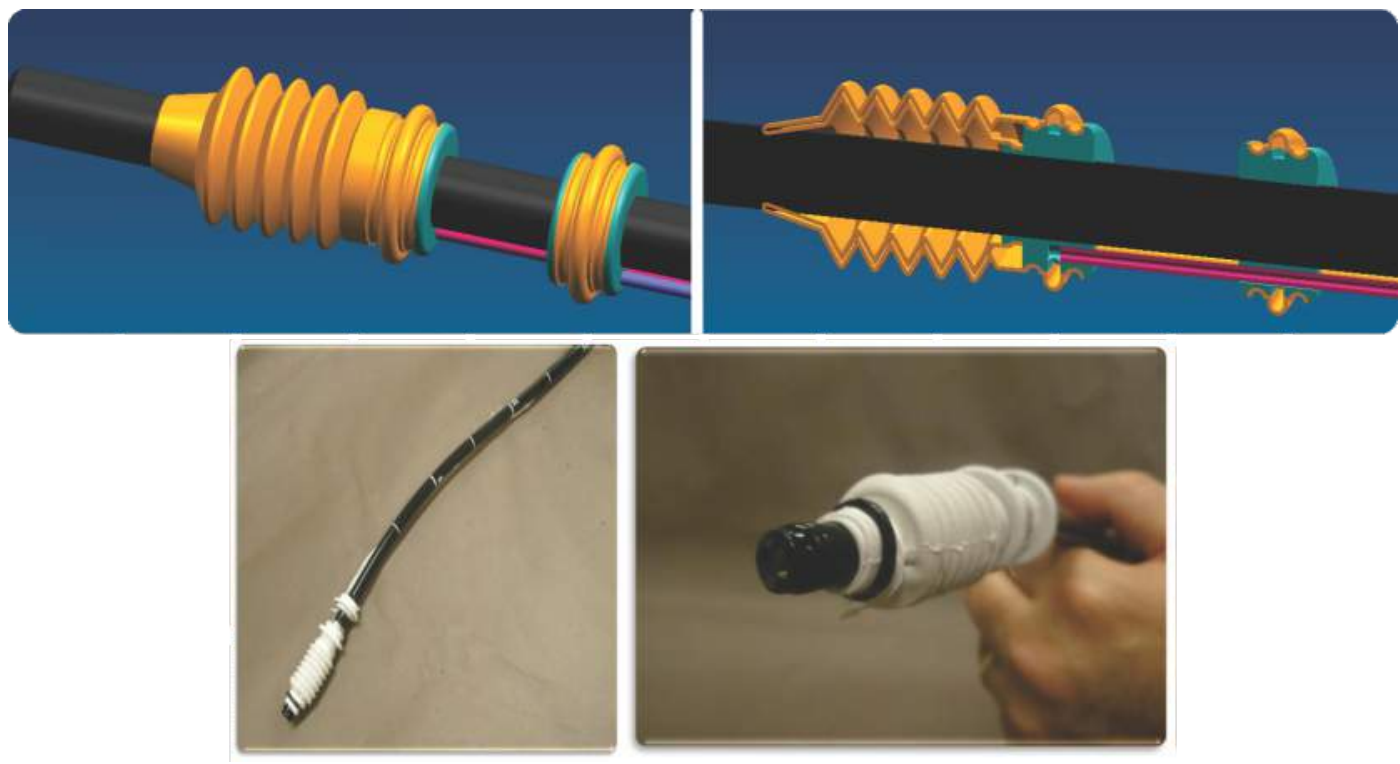

En noviembre de 2009, el resultado de la investigación fue presentado en el prestigioso congreso internacional especializado en endoscopia GASTRO y publicado en la revista indexada GUT.

El trabajo en equipo, en investigación del diseño, es necesario puesto que implica el contraste de ideas que enriquecen el trabajo y aportan diferentes niveles de innovación a las reflexiones, análisis y conclusiones. Especialmente en este trabajo, fue fundamental la interacción entre le equipo médico especializado en endoscopias, los diseñadores encargados de visualizar las ideas y construir los nuevos conceptos y los ingenieros de desarrollar los mecanismos de control. La revisión y constante evaluación de los pasos seguidos por cada uno de los equipos ha sido la garantía de los buenos resultados obtenidos.

El proyecto ha tenido repercusión en el Congreso Internacional GASTRO con la siguiente ponencia: Pons Beltrán, V.; Sanchez Diaz, C.; Santonja Gimeno, A.; Martinez Escapa, V.;Jorda Albiñana, B.; Songel Gonzalez, G.; Sala Felis, T. Results of the in vitro utilization of an automated device of movement (endoworm) adapted to a conventional enteroscopy, GASTRO 2009 UEGW/WCOG, London, 21-25 November.

El abstract de esta ponencia aparece recogido en:

- Gut, Nov 2009, Vol. 58 (Suplemento 2), pág. A45.

- Endoscopy, 2009, Vol. 41 (Suplemento I), pág. A45.

La revista GUT está indexada en la base de datos del JCR (SCI) en la categoría

ASTROENTEROLOGY \& HEPATOLOGY con un Factor de Impacto en el año 2008 de 9.766, ocupando el lugar 3 de 55 revistas en dicha categoría ordenadas las revistas por factor de impacto.

Además, está indexada en Index Medicus (Medline), ISI Current Contents (Web of Science) y Excerpta Medica (Embase). En 2008 su factor de impacto fue del 9.766 (2008 JCR Science Edition).

Otros datos: Periodicidad: Mensual + Suplementos. Inicio: 1960. ISSN: 1468-3288. Editor: Revista Oficial de la British Society of Gastroenterology.

La revista ENDOSCOPY es la revista oficial de la European Society of Gastrointestinal Endoscopy. Junto con GUT publica los abstract de UEGW en CD-Rom.

Otros datos: Periodicidad: Mensual. Inicio: 1961. ISSN: 0017-5749.

El dispositivo resultado del proyecto está sujeto a la patente internacional: WO/2009/098337:

Method and device for automated translational movement of an endoscope through the digestive tract.

La descripción de la patente reconoce que el dispositivo presentado supone un avance respecto a dispositivos similares porque permite un acceso más sencillo y eficaz al intestino delgado ya que el sistema es capaz de adaptarse a un endoscopio convencional y de replegar el intestino sobre el endoscopio, lo que facilita indirectamente el avance del mismo. En el funcionamiento del dispositivo son claves el diseño industrial y el diseño gráfico de la interfaz que permite la interacción entre médico y dispositivo. 


\section{[BIBLIOGRAFÍA CONSULTADA]}

BIEWENER, ANDREW. Animal locomotion. Oxford University Press. 2003

McNEILL, ALEXANDER. Principles of animal locomotion. Princeton University Press. Princeton 2003.

VOGEL, STEVEN. Comparative Biomechanics: Life's Physical World.

Princeton University Press. Princeton 2003.

WAINWRIGHT et alt. Diseño mecánico en organismos vivos. H. Blume. Madrid 1980.

\section{[7. EL FASCINANTE JUEGO DE LA NATURALEZA ] [25]}

La tradición en la educación entre el diseño y la naturaleza. Esta parte es una reflexión sobre la relación entre la naturaleza y los juguetes, y constituye la investigación básica, de la cual se derivarán los resultados en la fase de investigación aplicada y de desarrollo. Es también un breve recorrido por las culturas del mundo, por la vida de las personas, por la memoria de las sociedades, por la industria del entretenimiento, por las tradiciones didácticas, y todos esos ámbitos unificados por la observación de la naturaleza. Esta reflexión es resultante de la unión de las dos mayores creaciones que caracterizan al ser humano. Es decir, además de nuestra vida, y de la naturaleza que nos rodea, la interacción con la capacidad que más nos diferencia de los demás seres: el producir objetos para nuestra diversión. Esta recreación enfatiza la coincidencia entre la fascinante Naturaleza y los juguetes que transmiten la misma fascinación por la geometría, el crecimiento, la transformación, haciendo una invitación a la observación más profunda y respetuosa, un llamamiento a la capacidad inteligente de las personas para que descubran el origen de lo artificial en lo natural, o les permita intuir lo artificial a partir de lo natural. Sirva también para revalorizar aquellos juegos con el calificativo de educativos y que son menospreciados por la arrogante influencia medíatica de algunos fabricantes que pretenden hacerlos ver como necesariamente aburridos. Afortunadamente los juegos de construcción, aun siendo educativos, están demostrando ser muy activos en cuanto a las capacidades de construcción y transformación de los diferentes sistemas estructurales, y por lo tanto, en la adaptación a los requerimientos de los usuarios, en este caso los niños. Tres aproximaciones podríamos diferenciar en la relación entre la naturaleza y los juguetes. La primera de ellas sería la más ancestral, y posiblemente por ello la más universal en el tiempo y en el espacio: la naturaleza como materia prima del juego. La segunda correspondería a la más superficial, y posiblemente por ello más representativa de la sociedad de consumo actual, que convierte a la Naturaleza en objeto de juego, pero por lo menos impulsa y fomenta la capacidad de observación, aunque pasiva, del medio y los elementos que lo componen.

Posiblemente el mejor producto que represente esta categoría serían los hormigueros transparentes que permiten observar los recorridos de una pequeña comunidad de hormigas o las populares peceras. Sin embargo, recientemente los valores medioambientales se han convertido tanto en un requerimiento social globalizado y en una tendencia de mercado, y en consecuencia ha tenido su repercusión en el mundo educativo: asignaturas de educación medioambiental, políticas de recogida y tratamiento de residuos urbanos, políticas energéticas renovables, etc. La tercera aproximación representaría a los juegos y juguetes que transmiten la fascinación por la capacidad de crecimiento o transformación. Algunos se recrean en este aspecto, como por ejemplo los juegos de estructuras, otros inducen no solo a la observación de la fenomenología resultante, sino también a la indagación de los principios que llevan a tales manifestaciones. Subrayemos su contribución a recuperar la totalidad del valor educativo en cuanto a que ya no solo transmite un conocimiento, sino que genera una motivación y una actitud de constante aprendizaje. Habría una cuarta aproximación observable en cualquiera de las tres anteriores pero más difícil de medir porque entraría en la intimidad de todos y cada uno de nosotros y que correspondería al crecimiento paralelo del juego con la persona. Es decir, sería intentar medir la influencia que han ejercido algunos juegos o formas de jugar en nuestras vidas imprimiendo carácter o condicionando nuestra vida profesional. Dicho de otra forma sería medir el valor de juego por su duración a lo largo de la vida, que diferenciaría a los juguetes que pasan de temporada por su artificialidad, o aquellos que perduran por su universalidad.La naturaleza como materia prima del juego ha sido y sigue siendo una constante entre todos los niños de cualquier cultura y época. Jugar con granos de cereales o legumbres, construirse grandes cabañas con ramas de árboles o pequeños tirachinas con la misma rama, hacerse máscaras con calabazas secas o utilizar cáscaras de cocos como ruedas de arrastres estaría en la memoria colectiva de la infancia de muchas culturas y generaciones.

En cuanto a los juegos que transmiten la fascinación por la Naturaleza, creo necesario iniciar mencionando aquellos de tradición milenaria como los arcos entrelazados de la Orbe. No deja de sorprender estos caleidoscopios tridimensionales por su capacidad de transformación a través de 
unas semicircunferencias cuyos extremos giran a lo largo de dos circunferencias que siempre se mantienen paralelas.

Las formas que pueden adoptar irían desde la estructura radial plana que recuerdan a cualquier entramado artesanal de fibras naturales, hasta la esfera formada por pequeñas circunferencias. Aun más fascinante me parece el hecho de que en la propia tradición milenaria se incluya la explicación de la transformación del universo y sus componentes hasta convertirse en hombre, mujer y niños, a medida que se van transformando los aros y van apareciendo nuevas formas.

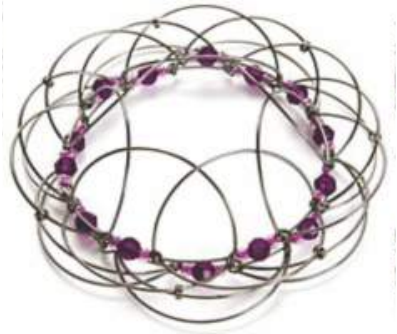

(imagen combinaciones ORB). Colección Gabriel Songel. Museo del Juguete UPV. Podríamos encontrar, por tanto, los antecedentes culturales de los juegos de estructuras en creaciones artesanales como el ORB o las creaciones en alambre y materiales reciclados realizados por niños africanos, que son perfectos ejemplos de libre creatividad y construcción expresiva. El modelado a base de unir alambres genera un sistema estructural único como un dibujo en tres dimensiones.
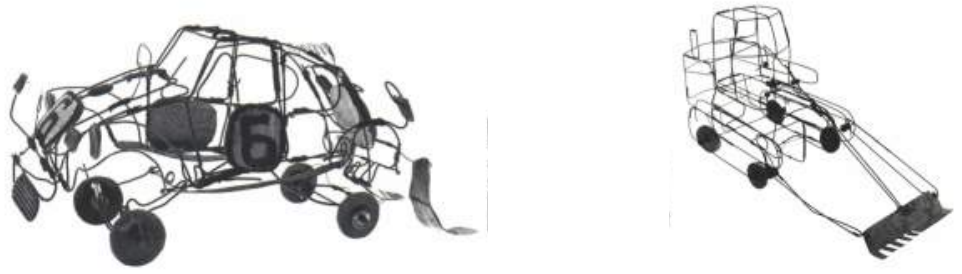

\section{[African structural toys. Colección Gabriel Songel. Museo del Juguete UPV]}

Los juegos de construcción son sin duda el origen de los juegos de estructuras. La principal diferencia reside en que, mientras en un juego de construcción las partes se apilan sobre sí mismas sin connexión, en los sistemas estructurales las uniones condicionan el volumen resultante a causa de la geometría y los angulos de sus partes. Por lo tanto, todos los juegos de estructuras son, a la vez, de construcción, pero no todos estos se pueden considerer estructurales. Y, evidentemente, no todos los juegos estructurales han tenido éxito en el mercado.

Meccano fue probablemente el primer sistema de construcción con tornillos y tuercas como union, pero fue el Tinkertoy el primer juego estructural. El sistema Meccano fue inventado por Frank Hornby en 1901 y su primer título para la invención fue "Mechanica hecha fácil" . Aunque Hornsby no era un ingeniero, su objetivo era enseñar los principios de ingeniería a niños. (1)

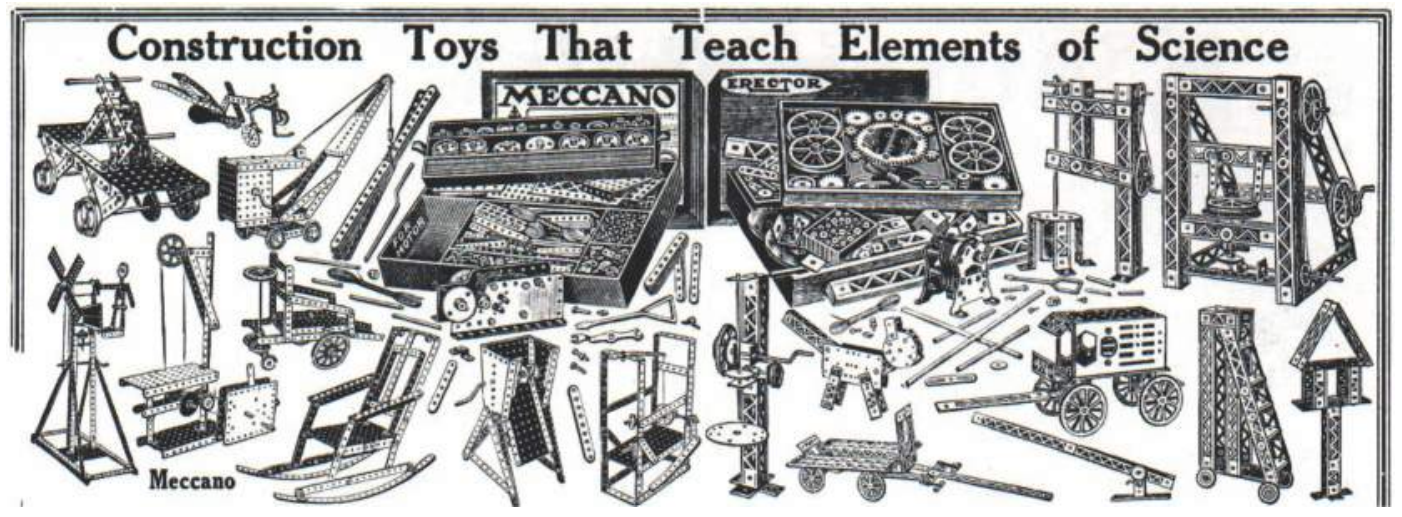

(picture 2. Old meccano. From Catalogue Sears.Roebuck and Co. 1921 in Collective Toys and games of the twenties and thirties from Sears Roebuck and co. Catalogs, Dover Publications New York1988) (2) 
Algo interesante a resaltar en este caso es la importancia de la cultura de la tecnología y su influencia en el dia a dia, algo apreciable hasta la actualidad. El hecho que las estructuras de hierro eran la tendencia tecnológica aplicada a la construcción de edicficios y puentes en el siglo XIX propiciaron la oportunidad de crear un producto commercial vigente hasta hoy en día en el mercado. Y no solo eso, sino que se ha convertido en un fenómeno social con clubes de coleccionistas, revistas especializadas y asociaciones internacionales. Pero fue el Tinkertoy el primer juego de estructuras que todavía se produce por la empresa norteamericana Hasbro. Tinkertoy fue introducida en Europa en 1924, aunque ya apareció en el catálogo de Sears de Chicago del año 1922. El sistema se basa en un disco grueso de Madera con ocho agujeros en la cara redonda y un agujero en la parte plana, donde se pueden conectar barras de madera.

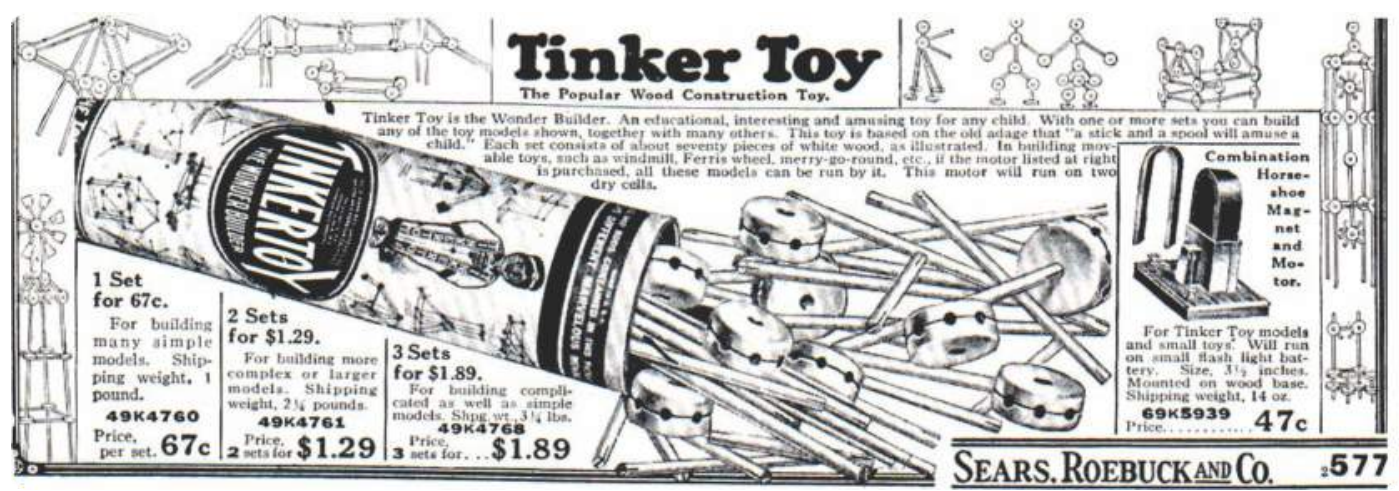

picture 3: Old tinker-toy. From sears...

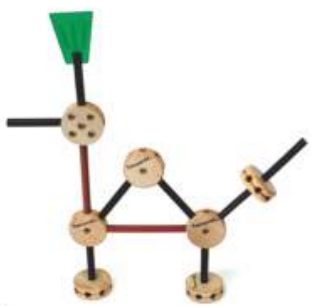

picture 4: tinker-toy. Colección Gabriel Songel. Museo del Juguete UPV

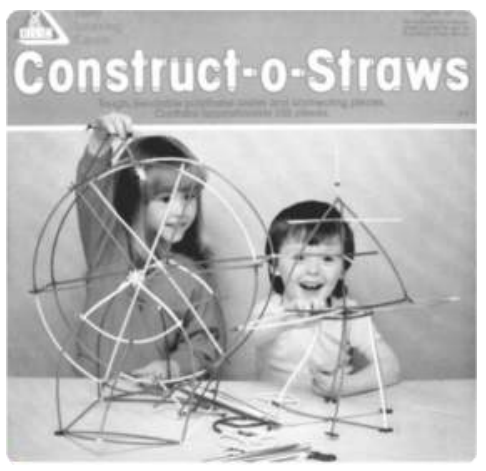

Construct-o-straws fue el primer sistema structural en la era del plástico introducida en el mercado como un juego educativo por Early Learning Center en el Reino Unido. El principio de economía y optimización de cualquier estructura fue rigurosamente aplicado como un requerimiento de diseño industrial que le hizo aparecer como un producto muy económico desde los años 60. La versión contemporánea de esta capacidad de transformación sería la esfera de Hoberman que se expande hasta 30 veces su volumen en contracción.

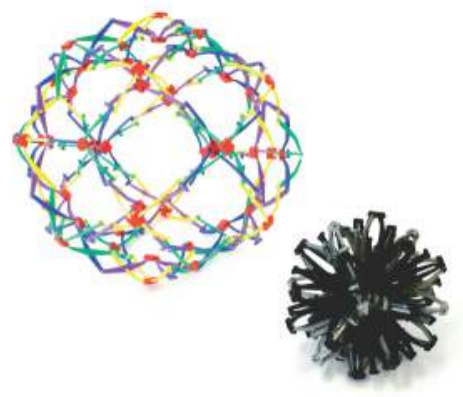

Hoberman se ha especializado en estructuras desplegables, habiendo llegado a trabajar para la NASA. Su esfera desplegable está fundamentada en que las aristas son pequeñas tijeras curvadas 10 grados que, desplegadas, forman triángulos y cuadrados intercalados, cuyos encuentros son los puntos de articulación. Actualmente una enorme esfera desplegable de aluminio construida por Hoberman esta en constantemente abriéndose y cerrándose en el atrio del Liberty Science Centre de Nueva Jersey. (imagen esfera desplegable). Colección Gabriel Songel. Museo del Juguete UPV. 


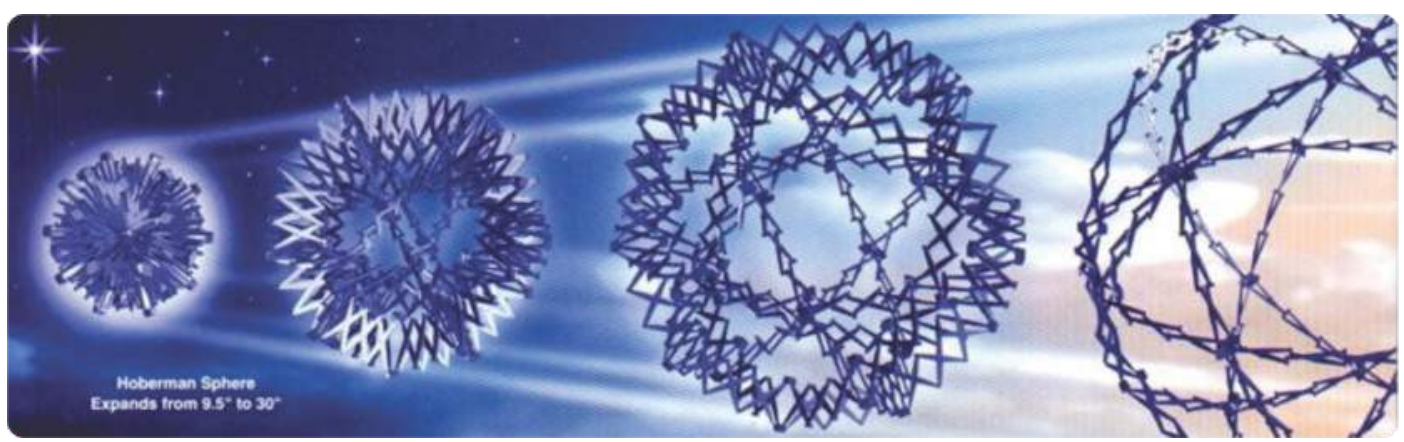

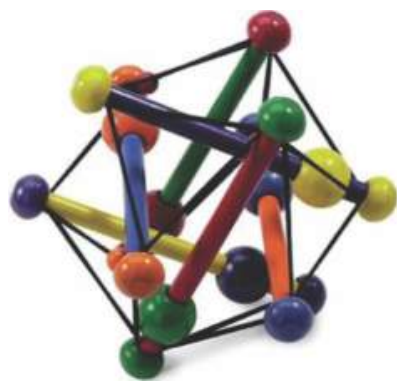
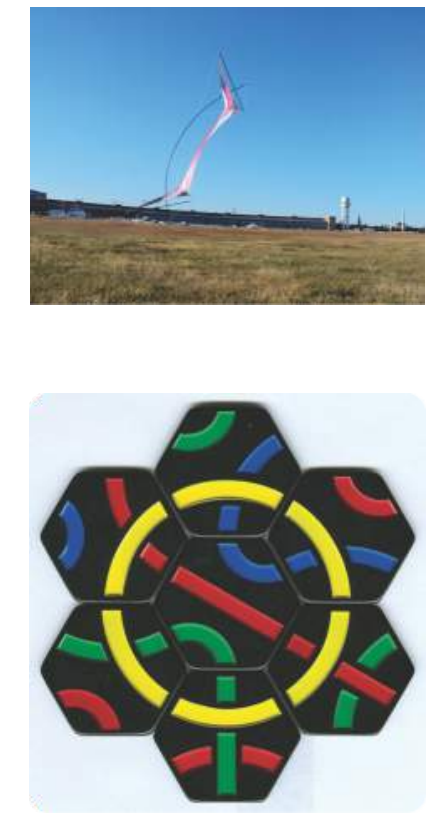

Siguiendo con las estructuras en tensión y retomando otro juego multicultural y ancestral como son las cometas, cabría mencionar las ultraligeras hojas volantes para interior de Zero Wind Kites diseñadas por Marc Ricketts. En realidad la fuerza del viento la da el jugador con ligeros movimientos de brazos o simplemente andando.

Pero Hoberman, en realidad es la última generación de diseñadores de estructuras. Y obviamente no es coincidencia que todos los pioneros del diseño de estructuras hayan sido aficionados a la observación de la geometría en la naturaleza. Los mismos principios de estructuras en tensegridad de Buckminster Fuller han sido aplicados a pequeños juegos de construcción Tensegrity - Stringlix.

\section{( Imagen Tensegrity - Stringlix.) Colección Gabriel Songel. Museo del Juguete UPV}

Las taselaturas islámicas podríamos decir que han sido redescubiertas gracias a que la geometría fractal se puso de moda hace veinte años. También las descubrió anteriormente el holandés Maurits $C$. Escher, al cual debemos mencionar por lo menos, por sus investigaciones sobre la modulación en constante mutación que tan interesantes e intrigantes juegos visuales dejó en su obra artística. Uno de los 23 problemas que dejó planteados el matemático Hilbert al inicio del siglo $\mathrm{XX}$, fue recisamente el de determinar de cuantas formas diferentes puede rellenarse por completo un plano con figuras geométricas idénticas. Igualmente habría que recordar que, años después, se demostró que solo había 17 formas posibles y están ejemplificadas todas ellas en la decoración de la Alhambra. Es sabido que Escher fue estimulado por la visita al palacio granadino e influenció a muchas de sus obras. Algunas de sus reflexiones en 1942 se plasmaron en la modulación para los Ornamentos I y II, que no es ni más ni menos, el juego de laberintos llamado Tantrix y conocido por el cubo Rubik de los 90 .

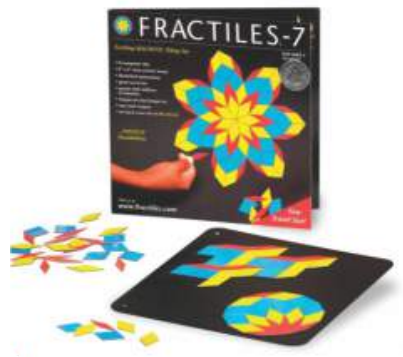

Otro buen ejemplo de juegos de taselaturas en la versión bidimensional serían los Fractiles magnéticos que con tres módulos rombos permite generar complicadas mallas, y que en su forma tridimensional constituye el sistema constructivo patentado por Haresh Lalvani. (imagen).

La versión tridimensional la representaría el sistema constructivo Polydron. Los diferentes polígonos tienen un punto de encuentro que les sirve tanto para unirse como para girar, de forma que induce a 
explorar la continuidad por los laterales de cualquier polígono hasta dar con las combinaciones que permitan cerrar toda la estructura. Evolución de Geomag y la geometría intuitiva a través de uniones imantadas.
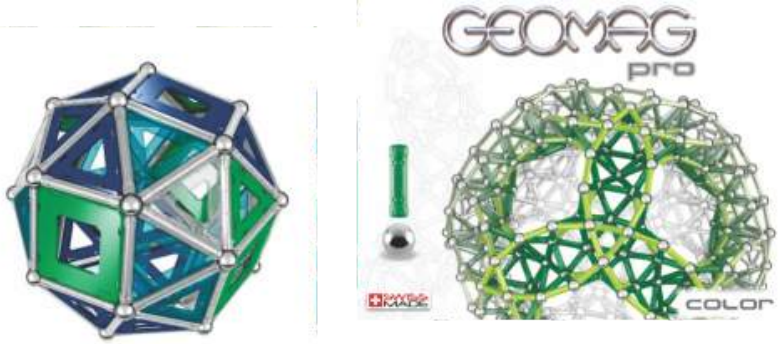

No podríamos hablar de juegos educativos geométricos y Naturaleza sin mencionar a Friedrich Froebel, conocido especialmente por ser el iniciador de los jardines de infancia o kindergarten a mediados del siglo XIX. En su filosofía de educación para los niños estaba la concepción de que el ser humano era "una parte y un todo". Los niños deberían desarrollarse como plantas en un jardín, siguiendo su propia naturaleza, pero observando la armonía con la totalidad de la naturaleza, de todo el universo. Sus métodos educativos intentaban transmitir al niño un sentido de unidad sistemática que fundamenta al mundo. Para ello inventó una serie de juegos geométricos que son las arquitecturas de letras y formas geométricas puras existentes actualmente en el mercado, así como los juegos de manualidades de modelar, coser, apilar bloques o plegar papel. Aunque María Montessori recoge algunos principios de Froebel pero dirigiéndolos hacia la psicología del comportamiento y generando su propio material didáctico y de experimentación, no es hasta Piaget y el conocimiento de los estadios del desarrollo de los niños, cuando se revaloran los juguetes para la primera infancia. Este campo es propicio para la experimentación formal abstracta y, por lo tanto, especialmente idóneo para la aplicación de conceptos de diseño básico. Así lo entendió Kurt Naef hace 40 años y desde entonces nos ofrece auténticos ejercicios de diseño básico en su catálogo con una especial atención en las formas del crecimiento. (imágenes)

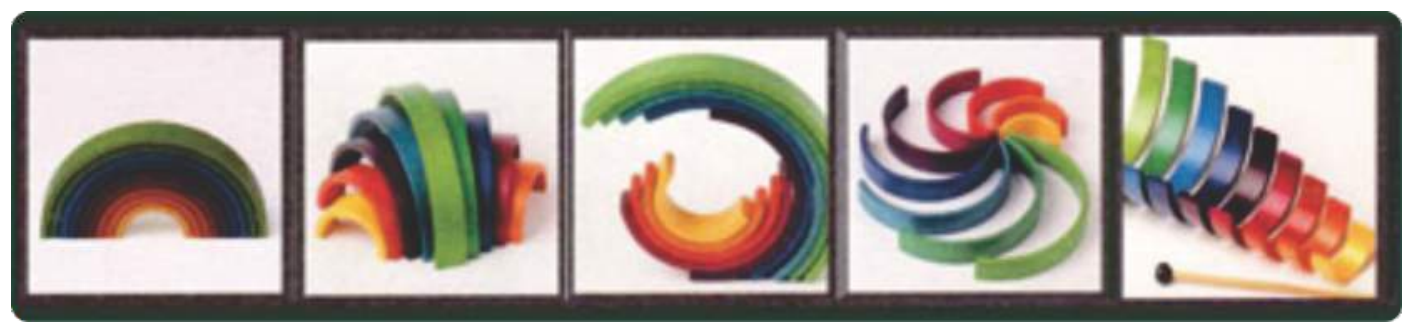

De entre los juegos de construcción, se podrían diferenciar los juegos de estructuras porque son los que mejor transmiten esa fascinación por la geometría y el descubrimiento de las formas de la Naturaleza. Sin embargo dentro de esta subcategoría - los juegos de estructuras - hemos visto una importante evolución en el desarrollo de nuevos sistemas constructivos que se adaptan a los requerimientos de los nuevos usuarios. Y es que, efectivamente, los juegos de estructuras ya no pueden ser solo ladrillos que se acumulan o barritas que se unen con tornillos y tuercas. Tampoco es ya suficiente que el sistema estructural sea tan complejo que aun permitiendo muchas posibilidades combinatorias reproduzca solo formas abstractas.

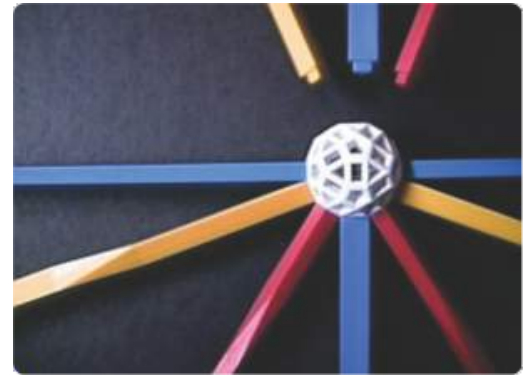

La enorme preocupación y conciencia de estas limitaciones de los grandes fabricantes de juegos de estructuras, ha sido la de conseguir sistemas flexibles, fácilmente transformables. Se han visto sistemas constructivos de geometrías muy complejas y de amplias posibilidades, pero, precisamente por ello, muy difíciles para jugar el niño que tiene que adivinar la geometría adecuada, buscar el ángulo correcto para poder cerrar la estructura, y eso genera frustración al niño y rechazo no solo hacia el juguete, sino a la forma de jugar. Tales serían los ejemplos de Zometool cuyo nudo estructural es un auténtico microorganismo esférico de 62 caras a las que se pueden conectar traviesas, o el Lock System de 26 caras.

zometool. Col. Gabriel Songel. Museo Del Juguete Upv

De hecho fue un gran avance cuando apareció el sistema K'nex que facilitaba el montaje por la forma de ensamblar las piezas e identificarlas por colores. Pero el problema persistía al no poder, o estar limitado el niño cuando al querer construir por su cuenta tiene que buscar el ángulo adecuado para compactar toda la estructura. Otro avance supuso la aparición del sistema Zoob fundamentado en un 
sistema de rótulas que se ensamblan unas con otras que permite realmente la articulación de los componentes pero sin llegar a ser consistentes en cuanto se agranda el volumen de la construcción. (imagen) Sin embargo la imagen del producto si que comunica ese aspecto orgánico que el propio eslogan dice Zoob: cuando la zoología se encuentra con la botánica.

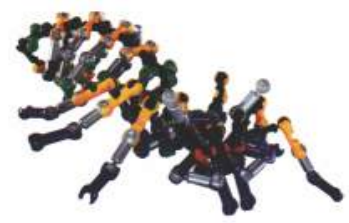

\section{ini?}

[REFERENCIAS BIBLIOGRÁFICAS]

SONGEL, G. et alt. Tendencias en el sector de ocio 2005. Ibi, Instituto Tecnológico del Juguete AIJU. 2005.

SONGEL, G., GARCIA-PROSPER,B . Identificación de tendencias en el sector juguetero. Ibi,

Asociación Española de Fabricantes de Juguetes, 2009.

SONGEL, G., GARCIA-PROSPER,B. Spora tendencias para juguetes. Ibi, Asociación Española de Fabricantes de Juguetes, 2011.

SONGEL, G et alt. El Museo del Juguete. Editorial UPV Universidad Politécnica de Valencia. 2004

Grundfeld, Frederic. Juegos de todo el mundo. Edilan. Madrid 1978.

Netz, Reviel y Noel, William. El código de Arquímedes. Temas de hoy. Madrid 2007

\section{[8. PROYECTO PINOS MÁGICOS]}

En homenaje al país organizador de esta publicación y su editor, quisiera mencionar el proyecto de juegos de construcción realizado para la empresa Elka de Brasil en 2014. El proyecto inició un año antes cuando fui invitado a la feria de juguetes ABRINQ en Sáo Paulo a dar una conferencia sobre tendencias de diseño de juguetes. La empresa Elka contactó entonces conmigo para realizar colaboraciones en el desarrollo de nuevos productos. La sorpresa fue descubrir el sistema de construcción Pinos Mágicos y la tradición de varias generaciones de niños y niñas brasileños que habían jugado con estas piezas de plástico de aspecto muy orgánico. Creo que es un buen ejemplo de

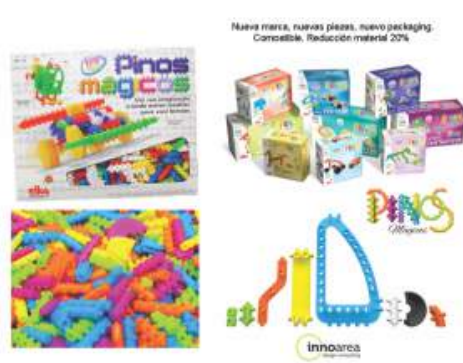
proyecto de diseño sostenible en su concepción mas amplia, ya que se trataba de respetar la cultura autóctona del diseño, con un producto ya conocido y muy asentado en la sociedad brasileña, por lo que el desarrollo de un nuevo sistema debía de tener en cuenta todas estas premisas sociales y culturales. Nuestro trabajo fue diseñar piezas nuevas que permitiesen mayor número de combinaciones y rediseñar las clásicas de manera que todas las piezas nuevas fuesen compatibles con las antiguas. Los Pinos Mágicos se fundamentan en un cilindro que en sus laterales tiene líneas de pinchos y líneas de agujeros. Entrando en la ingeniería de la inyección de plástico vimos que el molde era muy antiguo y que se podía optimizar la inyección. Conseguidos darle una pared más homogénea en toda la pieza y conseguimos ahorrar un $20 \%$ la masa del plástico, por lo que suponía un ahorro importante de materia prima. Desarrollamos también una propuesta de nueva marca, nuevos modelos con figuras orgánicas y nuevos packaging que fueron presentados en ABRINQ 2014. Un producto, por tanto, sostenible en el tiempo, en lo cultural, en lo material y en lo social.

\section{[9. PROYECTO GEOMETRÍA ROTATORIA]}

La investigación y experimentación de modelos de geometría rotatoria se manifiesta, entre otras formas, con la articulación de las cadenas de prismas geométricos. Ha sido una constante en la investigación básica de las artes plásticas, el diseño y la arquitectura. Los modelos de geometría rotatoria siempre se habían hecho en papel y con el Dinacube de Imaginarium conseguimos realizar una cadena articulada que plantea composiciones gráficas con las hay que adivinar la combinatoria y rotación de los módulos para conseguir esos patrones.
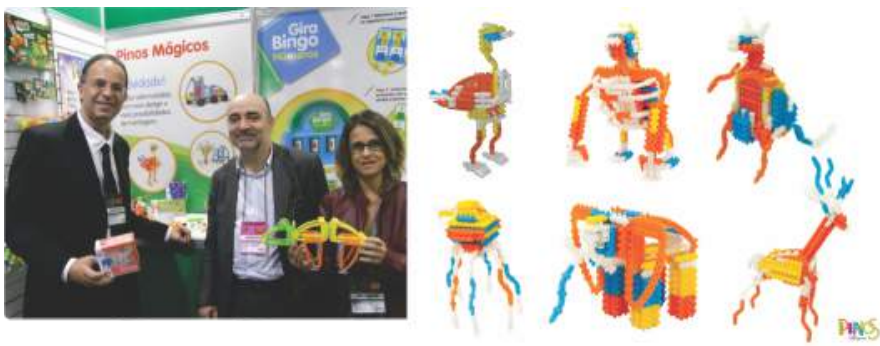
Dinacube. Gabriel Songel. Imaginarium 2013. Investigación en modelos de geometría rotatoria. Desde 2017 la empresa holandesa Recent Toys ha introducido de nuevo este producto para todo el mundo con nuevas presentaciones y efectos ópticos.
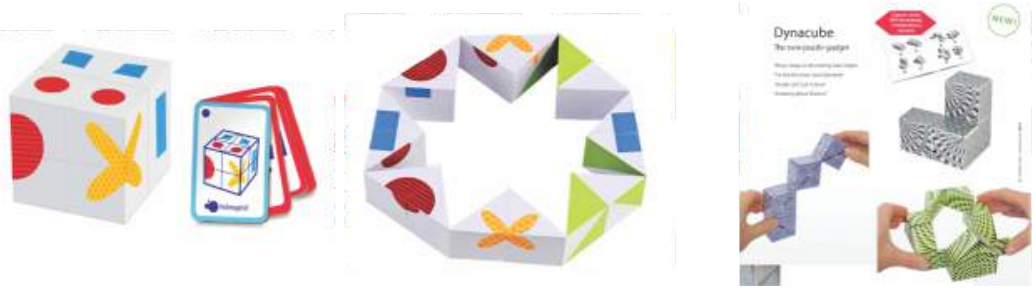

[1] Ignace Gelb. La historia de la escritura. Alianza editorial. Madrid

[2] Signos, Simbolos, marcas y señales. Gustavo Gili. Barcelona

[3] Paul Klee. Teoria de la forma y la representación.

[4] Paul Klee. Teoría del arte moderno. Cactus, 2007

[5] Christopher S. Henshilwood et al, An abstract drawing from the 73,000-year-old levels at Blombos Cave, South Africa, Nature (2018). DOI: 10.1038/s41586-018-0514-3

[6] AA.VV.Temes de Disseny: Naturaleza Diseño e Innovación. Escuela Elisava. Barcelona, 1994.

[7] Von Bertalanffy, Ludwig, Perspectivas en la teoría general de sistemas, Alianza Universidad, Madrid, 1982 , p. 95.

[8] Pearce, Peter, Structure in Nature is a Strategy for Design, MIT Press, Cambridge, 1978. Stevens, Peter, Patrones y pautas en la naturaleza, Biblioteca Científica Salvat, Barcelona, 1986.

[9] Di Bartolo, Carmelo, Strutture Naturali e Modelli bionici, Departamento of Industrial Design, Istituto Europeo di Design, Milán, 1981

[10] Lecuona, M., y Songel, G., Design Research and Design Management, Ponencia en el Fifth International Forum on Design Management, Research and Education, Sloan School of Management, MIT Boston, 1993.

[11] Songel, Gabriel, Estudio metodológico de la biónica aplicada al diseño industrial, tesis doctoral, ETSI Industriales, Universidad Politécnica de Valencia, mayo de 1991.

[12] Otto, Frei, Natürliche Konstruktionen, Deutsche Verlaganstalt, Stuttgart, 1982, p. 8.

[13] Coineau, Yves, y Kresling, Biruta, Les inventions de la Nature et la bionique, Hachette, París, 1987, p. 7.

[14] Nachtigall, Werner, La Nature réinventée, Pión, París (Allain Bougrain-Dubourg), 1987, p. 9.

[15] Gerardin, Luden, La bionique, Hachette, París, 1968. Mironov, Igor, La bionique, Mir, Moscú, 1970, p. 14.

[16] Papanek, Viktor, Diseñar para el mundo real, Hermann Blume, Madrid, 1977, p. 191

[17] Bonsiepe, Gui, Teoría y práctica del diseño industrial, Gustavo Gili, Barcelona, 1975, p. 132.

[18] Di Bartolo, Carmelo, conferencia en Encuentros de Diseño, Impiva, Alicante, julio de 1985.

[19] Nachtigall, Werner, op. cit., p. 48.

[20] Coineau, Yves, y Kresling, Biruta, op. cit., p. 7.

[21] Ibid.

[22] Temes de disseny, 1994.

[23] AA.VV. El juego para todos en los parques infantiles" AIJU, Instituto del Juguete y afines. Ibi (Alicante) 2001

[24] WAINWRIGHT et alt. Diseño mecánico en organismos vivos. H. Blume. Madrid 1980

\section{[10. CONCLUSIONES]}

Se ha presentado una serie de reflexiones sobre la implicación de la naturaleza y el diseño y la innovación. Y aunque, en un primer momento se recurría al entorno natural como un recurso más de innovación, hemos ido dándole forma hasta darnos cuenta que la Naturaleza tiene su propia estrategia, de la misma manera que profesionalmente nos hemos tenido que ir adaptando a los diferentes proyectos que hemos desarrollado. Estos han sido de dos tipos: unos de iniciativa propia, desarrollados hasta niveles conceptuales que luego han sido implementados por las empresas que los han comercializados, y otros por demanda de los interesados que nos lleva a seguir tiempos y requerimientos propios de mercado. Sin embargo unos y otros han conllevado desarrollar un conocimiento y una experiencia que nos ha llevado a otras oportunidades. Este discurrir de la experiencia profesional, imposible de planificar por las vicisitudes de la propia vida, si que nos han ido abriendo puertas a otros niveles impensables de que sucedieran años antes. Creo que esta la verdadera estrategia de la Naturaleza: el trasvase del conocimiento, como la savia que surge de las raíces (lo que somos) hasta el último sarmiento (el último proyecto) en cada ciclo de nuestra vida. Acumulación de conocimiento, transformación en energía y capacidad de adaptación son virtudes de la Naturaleza que tienen su analogía en la vida profesional. La acumulación de conocimiento, por si no tiene mayor interés, ya que solo ocupa mucho espacio. Lo estimulante y permanentemente enriquecedor es la constante conversión del conocimiento en nuevas ideas cuya implementación generará nuevas experiencias y nuevo conocimiento. La capacidad de adaptación es mera ley de subsistencia ya que no podemos dirigir nuestra vida como nos gustaría, sino en función de las oportunidades que nos busquemos o que nos ofrezcan. Afortunadamente, así lo vivo y lo creo, la visión retrospectiva de nuestra vida, nos permite ver que hasta los errores que hayamos podido cometer, han tenido un sentido positivo para nuestro crecimiento como personas y como profesionales. Si además nos dedicamos a la docencia, creo que la mejor forma de transmitir conocimiento y experiencia, es transmitiendo la emotividad y el convencimiento, la pasión y el saberhacer acumulado. Descendiendo al nivel del que quiere aprender y encendiendo su motor de curiosidad, desarrollando la transmisión de la motivación, llevándolos a la concreción de las ideas, sopesando su impacto en la sociedad y en medio ambiente, llegaremos a formar personas capaces de cambiar el mundo. Esa es la estrategia de la innovación en la Naturaleza. 